\title{
Laboratory experiments with tilted convective plumes on a centrifuge: a finite angle between the buoyancy force and the axis of rotation
}

\author{
By VITALII A. SHEREMET ${ }^{1} \dagger$ \\ ${ }^{1}$ Department of Physical Oceanography, Woods Hole Oceanographic Institution, \\ Woods Hole, MA 02543, USA
}

(Received 20 March 2001 and in revised form 11 December 2003)

The effect of both vertical and horizontal components of the Earth's rotation on plumes during deep convection in the ocean is studied. In the laboratory, the misalignment, characterized by the angle $\alpha$, between the buoyancy force ('effective' free-fall acceleration $\boldsymbol{g}_{e}$ ) and the rotation axis $\boldsymbol{\Omega}$ is produced by using the centrifugal force: an experimental tank was placed at a large distance from the centre of the turntable. The mathematical analogy between the laboratory model and the oceanic environment is presented. For $\alpha=30^{\circ}$, a number of laboratory experiments spanning a wide range of the buoyancy flux parameter, and correspondingly Reynolds number, is used to illustrate the development of the convective plume from a point source in regimes ranging from weakly to highly turbulent. New features of the flow, as compared to $\alpha=0$, are documented and explained.

The incoming heavier dyed fluid jet disintegrates into fast-sinking coherent blobs (in a low-Reynolds-number regime) or turbulent billows (in a high-Reynolds-number regime) and a more diffuse cloud of highly diluted dyed water. An analysis of the forces acting on an ellipsoid moving in a rotating fluid with the main balance including the buoyancy, Coriolis forces, and the hydrodynamic reaction due to generation of inertial waves correctly predicts the trajectory of a descending blob. It also explains the tendency of the plume to develop in the direction intermediate between $\boldsymbol{g}_{e}$ and $\boldsymbol{\Omega}$ and to shift 'eastward' (lagging the rotation of the centrifuge) if the plume is envisaged as an ensemble of blobs.

The stretching of the highly diluted dyed water along the absolute vorticity tubes with simultaneous shearing by horizontal quasi-two-dimensional flow produces conspicuous tilted structures or tilted Taylor 'ink walls'. The misalignment between $\boldsymbol{g}_{e}$ and $\boldsymbol{\Omega}$ enhances the turbulent mixing and development of tilted structures by breaking the symmetry and producing motions directed away from the rotation axis.

We argue that the conditions at the sites of ocean deep convection are favourable for the development of tilted structures because of the smallness of the Rossby number and an extreme homogenization of the mixed layer. We hypothesize that the homogenized sublayers observed within actively convecting regions in the ocean may not be horizontal, but in fact analogous to the tilted 'ink walls' observed in the laboratory experiments and that they represent the internal structure of a plume on horizontal scales smaller than its depth.

$\dagger$ Present address: Graduate School of Oceanography, University of Rhode Island, Narragansett, RI 02882, USA. 


\section{Introduction}

Convection in rotating fluids is a widely present natural phenomenon, occurring in stellar and planetary interiors, atmospheres and hydrospheres. It also occurs in many practical engineering applications involving centrifuges. In particular, our main motivation for the present work comes from the oceanographic phenomenon of deep convection. It is known to play a key role in the process of deep-water formation and driving the global ocean thermohaline circulation responsible for approximately half of the poleward transport of heat by the atmosphere-ocean system (Macdonald \& Wunsch 1996). Deep convection is restricted to only a few places in the world ocean, mostly at high latitudes in the Labrador, Greenland and Weddell Seas (see the review volume edited by Chu \& Gascard (1991) and also the review paper by Marshall \& Schott (1999) for a summary of all known deep convection sites). When and where it does occur, however, deep convection is a spectacular violent process capable of vertically mixing patches of water up to hundreds of kilometres across to a depth of one kilometre or more over a period of a few days. This process is very complex and is characterized by a number of spatial and time scales. As is currently known, three phases of deep convection can be identified (not always distinctly): preconditioning; violent mixing; and spreading of the denser water formed. Deep convection typically results in the formation of a cylinder of homogeneous dense water, with a diameter of 10 to $100 \mathrm{~km}$ and several kilometres deep, known as a 'chimney.' However, according to observations (Schott, Visbeck \& Fischer 1993; Vaughn \& Leaman 1995; Lilly et al. 1999), the actual vertical mixing is principally carried out by much smaller convective cells, or plumes, with horizontal scales of less than one kilometre, vertical velocities of up to $10 \mathrm{~cm} \mathrm{~s}^{-1}$ and time scales ranging from hours to days. Such convective plumes (as any other motions with time scales longer than several hours for which the Rossby number is small) will be strongly affected by the Earth's rotation. It is these convective plumes that are of primary interest here.

As demonstrated in the classical laboratory experiments in a rotating tank with the angular velocity $\boldsymbol{\Omega}$ parallel to the direction of acceleration due to gravity (Taylor 1921; Long 1954), the tracer evolution in a rotating environment has a very peculiar character due to the Coriolis force: fluid parcels with anomalous values of a tracer tend to be aligned along the axis of rotation. This is a consequence of the TaylorProudman theorem

$$
(\boldsymbol{\Omega} \cdot \nabla) \boldsymbol{v}=0
$$

stating that the gradient of the velocity field $v$ along the axis of rotation is vanishing or, in other words, when the rotation dominates, fluid motions tend to be restricted to vertical Taylor columns. The whole pattern has a very dramatic appearance resembling 'hanging curtains' and is called Taylor 'ink walls'. In the experiments mentioned above, an almost neutrally buoyant dye was injected into the homogeneous fluid. However, the formation of the 'ink walls' is mainly due to the vertical stretching of the initial intrusion along the absolute vorticity tubes and simultaneous shearing by a horizontal quasi-two-dimensional flow. The same mechanism will also work in the case of convection when the intrusion has a slight buoyancy anomaly.

In the ocean, the direction of the Earth's rotation $\boldsymbol{\Omega}$ is not collinear with the acceleration due to gravity $g$ (except at the poles); this has important consequences on convection. In a paper dealing with the slantwise convection in frontal regions, Straneo, Kawase \& Riser (2002) conclude that in the presence of a horizontal component of rotation, the turbulent mixing occurs in a slantwise fashion resulting 
in a different depth of the mixed layer. There is also indirect evidence that certain convective motions may be aligned along the axis of rotation rather than along the direction of acceleration due to gravity. The temperature-salinity records obtained from vertical CTD (a Conductivity-Temperature-Depth measuring probe used for standard hydrographic surveys) casts often reveal distinct homogenized sublayers 50$100 \mathrm{~m}$ thick separated by sharp steps within a well-mixed convective layer (Pickart, Torres \& Clarke 2002). The origin of these sublayers is currently unknown. Our hypothesis is that the convection tends to homogenize fluid in the direction of rotation within tilted Taylor 'ink walls' and not in the direction of the gravitational force. As a CTD travels vertically, it intersects the tilted structure of the temperature and salinity fields resulting in a detection of apparent sublayers.

The horizontal component of the Earth's rotation is usually neglected for largescale oceanic motions (a so-called traditional approximation of the Coriolis force) as a consequence of the shallow-water and hydrostatic approximations. These approximations are not valid in the case of deep convection: convecting columns may have an aspect ratio of unity, vertical velocities may be as large as the horizontal, and vertical accelerations cannot be neglected.

It is not trivial to model oceanic convection in the case with a finite angle $\alpha$ between the axis of rotation and gravitational force in the laboratory. There have been laboratory experiments on convection in a closed cylindrical tank rotating along its axis with the axis of rotation tilted with respect to the vertical direction (Boubnov \& Golitsyn 1995). However, such a configuration is essentially different from the oceanic environment: viewed in the reference frame rotating with the cylinder, the vector of the gravitational force will rotate describing a cone, hence on average the gravitational force will be collinear with the axis of rotation. Therefore, it is not surprising that in those experiments the convective motions were aligned along the axis of rotation.

To model properly in a laboratory the geophysically relevant case of a finite angle between the gravitational force and the axis of rotation, with both vectors fixed in the rotating reference frame, we can use a centrifugal force (see sketch in figure 1). The centrifugal force causes the free surface of the rotating fluid to assume the shape of a paraboloid. The total buoyancy force acting on a fluid parcel is the sum of gravitational and centrifugal forces; it is perpendicular to the fluid free surface, and therefore is not collinear to the vertical axis of rotation (see Fletcher 1972 for a discussion and experimental validation of the apparent field of gravity in a rotating fluid system).

The possibility of using centrifugal force was recognized long ago, and there have since been many laboratory studies of the effect of both components of rotation on convective motions (see the review paper by Busse et al. 1998). However, all such experiments were conducted in circular geometry (spherical shell, cylindrical annulus, paraboloid). These experiments were focused mostly on global scales of motion, and their results are applicable to zonal motions in stellar and planetary atmospheres, addressing the question of equator-to-pole heat transfer by large-scale circulation. We also note the difficulty in visualizing convective motions in these experiments owing to an oblique viewing angle and refraction.

To study the effect of both vertical and horizontal rotation components on convective motions and concentrate on scales comparable with the plume, we need to take a completely different experimental approach: we need to use a centrifuge an experimental tank should be placed at some large radius from the axis of rotation rather than in the center of a turntable. No experimental studies of convection (e.g. a rising or sinking plume) or the role of the horizontal component of rotation in such 
(a)

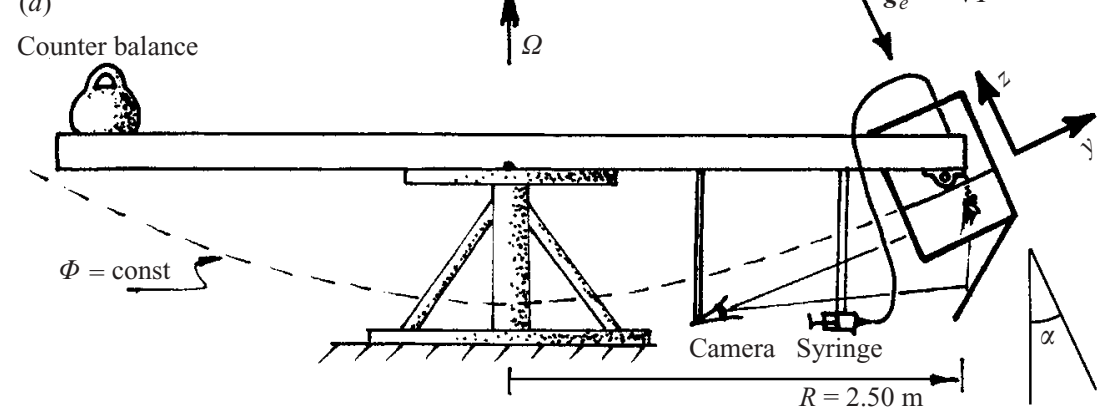

(b)

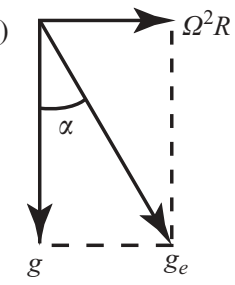

(c)

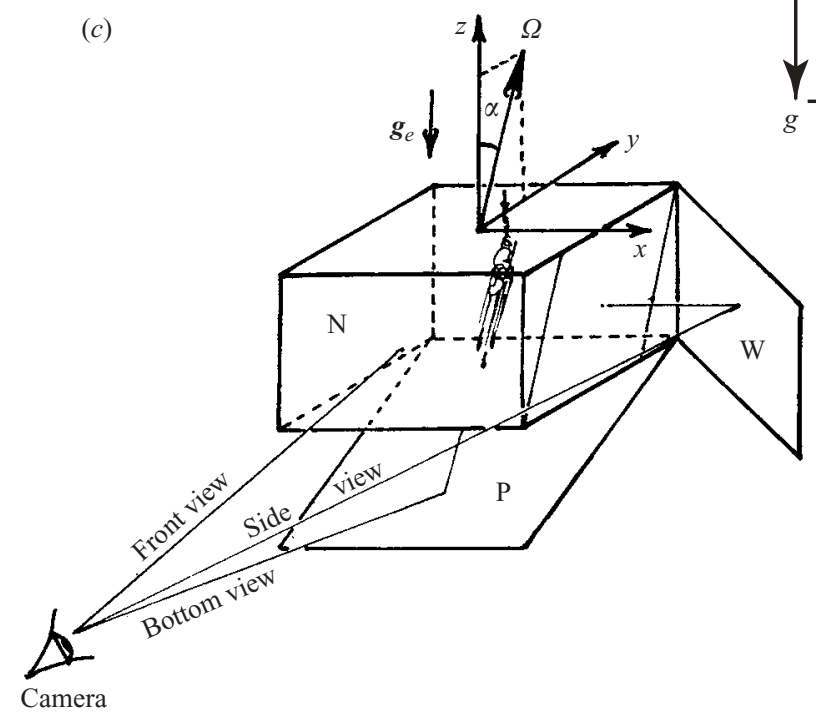

FIGURE 1. Sketch of the experimental design $(a)$ location of experimental devices, $(b)$ the effective gravitational acceleration vector diagram, $(c)$ capturing three simultaneous views.

localized settings are known in the oceanographic literature. The experimental work reported here is the first such study to date. We can think of the present experimental apparatus as a 'cutout' in the paraboloid configuration.

There is extensive literature on the so-called slantwise convection in the atmosphere and on a similar phenomenon in the ocean (for example, Emanuel 1988; Straneo et al. 2002). The well-established theory of slantwise convection is intended to explain the presence of mesoscale $(20-200 \mathrm{~km})$ bands within a cyclone's precipitation areas. It is based on the mechanism of a conditional symmetric instability. This means that locally the atmosphere may be statically stable, but because of the substantial shear of mean flow in areas with strong horizontal density gradient, instability can develop in a slantwise fashion along surfaces of absolute momentum. Formerly, the slantwise convection meant the baroclinic instability which is approximately geostrophic and hydrostatic: the horizontal scales of slantwise convection are much larger than the vertical ones. Here we study a completely different phenomenon: in essence, the 
effect of rotation on buoyant plumes. The differences are that the plumes themselves originate because conditions are locally unstable to vertical motions and the dynamics is neither hydrostatic nor quasi-geostrophic. To avoid confusion, we shall use the term tilted convection.

\section{Mathematical formulation of a laboratory model}

In a reference frame rotating at the angular velocity $\boldsymbol{\Omega}$, the Navier-Stokes equations governing the flow in a tank have the familiar form

$$
\begin{gathered}
\frac{\mathrm{d} \boldsymbol{v}}{\mathrm{d} t}+2 \boldsymbol{\Omega} \times \boldsymbol{v}=-\frac{1}{\rho} \nabla p+v \nabla^{2} \boldsymbol{v}-\nabla \Phi, \\
\nabla \cdot \boldsymbol{v}=0,
\end{gathered}
$$

where $v$ is the velocity vector and $p$ is pressure; $\mathrm{d} / \mathrm{d} t$ is the substantial time derivative; $\rho$ is the variable density of water, dependent mostly on salinity and slightly on the concentration of dye; and $v$ is the viscosity of water. The gravitational and centrifugal forces are combined in the potential

$$
\Phi=g h-\frac{1}{2} \Omega^{2} r^{2}
$$

where $h$ is the height measured vertically and $r$ is the distance from the axis of rotation. In a solid-body rotation state, the free surface of fluid (a surface of constant pressure $p$ ) will coincide with the surface of a constant potential $\Phi$ in (2.3); therefore, the local slope of the free surface is given by (see figure $1 a, b$ )

$$
\tan \alpha=\frac{\mathrm{d} h}{\mathrm{~d} r}=\frac{\Omega^{2} r}{g} .
$$

For example, if an experimental tank is placed at the radius $r=2.5 \mathrm{~m}$ and the acceleration due to gravity $g=9.803 \mathrm{~m} \mathrm{~s}^{-2}$, in order to achieve $\alpha=30^{\circ}$ in our laboratory we need to set the rotation rate of the platform $\Omega=1.509 \mathrm{~s}^{-1}$.

It is convenient to introduce a right Cartesian coordinate system $(x, y, z)$ associated with the tilted tank (figure $1 c$ ), with $z=0$ corresponding to the bottom of the tank, while $x, y= \pm L / 2$ corresponds to the sidewalls, which rotates relative to the laboratory reference frame $(r, \lambda, h)$ with the angular velocity $\Omega=\mathrm{d} \lambda / \mathrm{d} t$. The axes $(y, z)$ are in the same plane as $(r, h)$, but rotated inward through the angle $\alpha$. When the tilt angle $\alpha, \Omega$, and the radius $R$ at which the tank is located are matched according to (2.4), the free surface of the fluid will be approximately parallel to the bottom and, if we neglect its curvature, will correspond to $z=H$. The error of such an approximation according to $(2.3)$ is $O\left((L / R)^{2}\right)$; therefore, it is important that the size of the tank $L$ be much smaller than its distance $R$ from the axis of rotation. (This is analogous to ignoring the beta effect or variation of the Coriolis parameter with latitude.) In this coordinate system (as illustrated in figure $1 b, c$ ), the angular velocity will have two non-zero components

$$
\boldsymbol{\Omega}=\left(\Omega_{x}, \Omega_{y}, \Omega_{z}\right)=(0, \Omega \sin \alpha, \Omega \cos \alpha),
$$

while the acceleration due to the gradient of the potential will have only one non-zero $z$-component

$$
-\nabla \Phi=\left(-\frac{\partial \Phi}{\partial x},-\frac{\partial \Phi}{\partial y},-\frac{\partial \Phi}{\partial z}\right)=\left(0,0,-g_{e}\right),
$$


where

$$
g_{e}=\sqrt{g^{2}+\left(\Omega^{2} R\right)^{2}}=g / \cos \alpha
$$

is the 'effective' free-fall acceleration.

Written in this coordinate system, the momentum equations (2.1) in terms of velocity components $\boldsymbol{v}=(u, v, w)$

$$
\begin{aligned}
\frac{\partial u}{\partial t}+(\boldsymbol{v} \cdot \nabla) u+2\left(\Omega_{y} w-\Omega_{z} v\right) & =-\frac{1}{\rho} \frac{\partial p}{\partial x}+v \nabla^{2} u, \\
\frac{\partial v}{\partial t}+(\boldsymbol{v} \cdot \nabla) v+2 \Omega_{z} u & =-\frac{1}{\rho} \frac{\partial p}{\partial y}+v \nabla^{2} v, \\
\frac{\partial w}{\partial t}+(\boldsymbol{v} \cdot \nabla) w-2 \Omega_{y} u & =-\frac{1}{\rho} \frac{\partial p}{\partial z}+v \nabla^{2} w-g_{e},
\end{aligned}
$$

have exactly the same form as equations for the oceanic flow on an $f$-plane with $x$ directed eastward, $y$ northward, $z$ upward, and with $g$ replaced by $g_{e}$. The tilt angle $\alpha$ should be interpreted as the complement to the geographical latitude $\varphi=90^{\circ}-\alpha$, while $\Omega_{z}, \Omega_{y}$ should be interpreted as the vertical and horizontal components of the Earth's angular velocity, respectively. We shall use these 'geographic' analogues to specify unambiguously which side or view (see figure $1 c$ ) of the laboratory tank we refer to. We emphasize that the meanings of 'north, east, etc.' are based only on the similarity of the form of the equations, which might be counterintuitive for some readers who associate 'north' with the direction to the center of turntable and 'east' with the direction of rotation of the centrifuge. In our notation, 'north' $(y)$ is outward and 'east' $(x)$ is in the direction opposite to the rotation. The latitude of the Labrador Sea convection site $\varphi$ is about $60^{\circ}$ corresponding to $\alpha=30^{\circ}$ in the laboratory, which was fixed for all our experiments.

In the laboratory, we cover the plume dynamics regimes ranging from weakly to highly turbulent. For example, the typical vertical velocities $w$ were observed to range from $0.04 \mathrm{~cm} \mathrm{~s}^{-1}$ to $2 \mathrm{~cm} \mathrm{~s}^{-1}$; therefore we infer that the Reynolds number $R e=w H / v$ varied from 80 to 4000 . At the high end of $R e$, the incoming plume had apparent three-dimensional turbulent billows characteristic of the well-developed turbulence and we assume that the turbulent cascades are roughly independent of $R e$ and are similar to the oceanic ones. Therefore, the key non-dimensional parameter that ensures the similarity between the laboratory experiment and the oceanic environment is the natural Rossby number (Marshall \& Schott 1999)

$$
\epsilon=\frac{w}{2 \Omega H},
$$

where $w$ is a characteristic vertical velocity of convective motions. For the rotation to play a role in convection, $\epsilon$ must be $O(1)$ or smaller. As mentioned in $\S 1$, time scales for the oceanic convective plumes are from several hours to days; therefore this condition is satisfied in the ocean. Our laboratory experiments satisfy this condition without any difficulty and span a wide range of $\epsilon$ from $6.6 \times 10^{-4}$ to $3.3 \times 10^{-2}$.

\section{Experimental apparatus}

In order to study tilted convection, an experimental centrifuge was designed and built (see figures $1 a$ and 2). It consisted of a highly stabilized and reinforced rotating platform with a wooden frame extending from the centre $2.5 \mathrm{~m}$ on each side. A box-shaped experimental tank had inner horizontal dimensions $L \times L(L=29 \mathrm{~cm})$ 

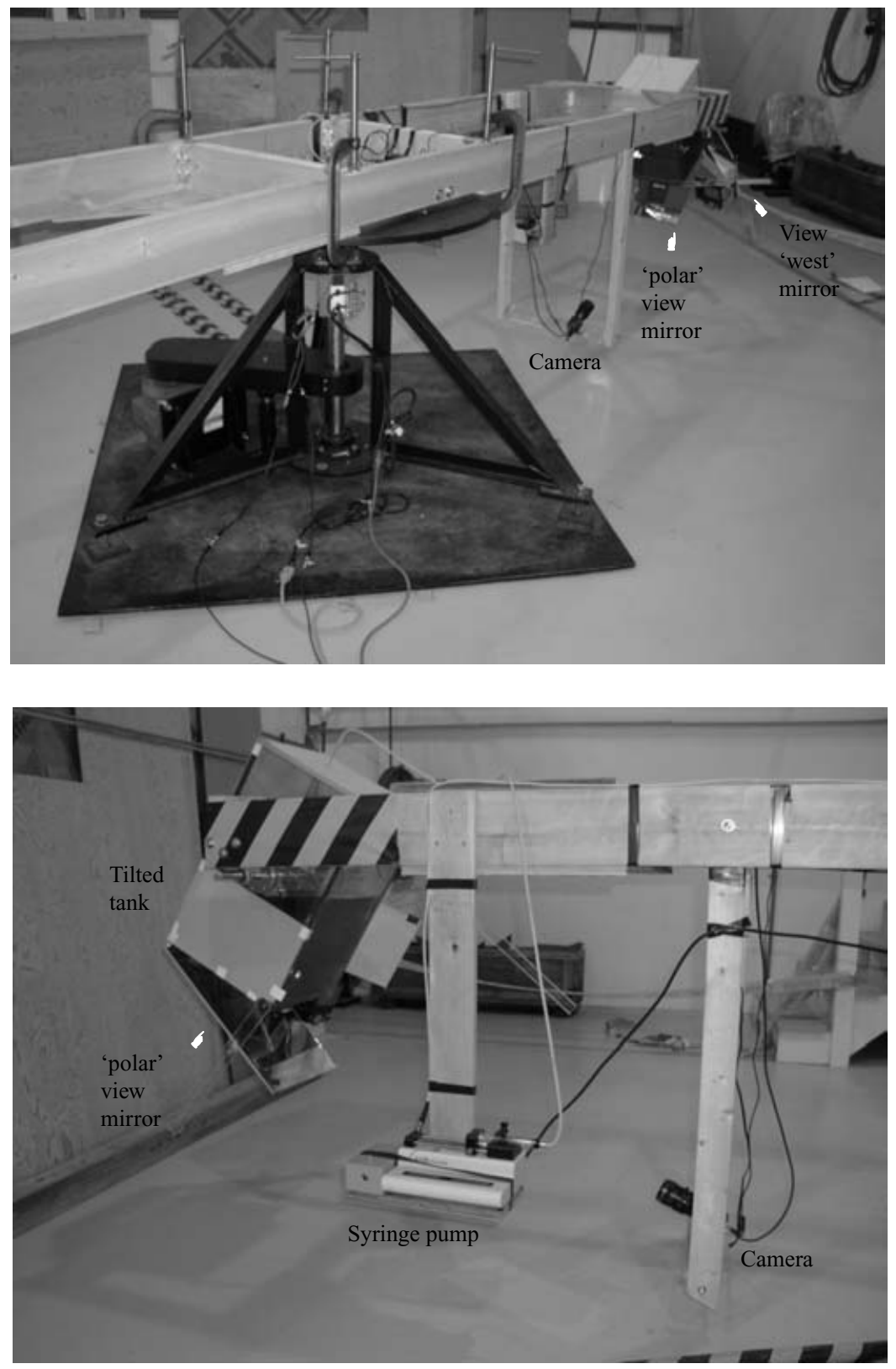

Figure 2. (a) The fully assembled experimental centrifuge. (b) A photograph zoomed on a tilted experimental tank, two mirrors providing two additional views, a syringe pump and a video camera.

and height of $50 \mathrm{~cm}$. It was attached at one end of the wooden frame by two pivots allowing the tank to be tilted inward. All experiments reported in this paper were conducted with the tilt angle preset at $\alpha=30^{\circ}$. The rotation rate of the platform $\Omega=1.509 \mathrm{~s}^{-1}$ was matched according to (2.4) so that the free surface of the fluid in the tank (which is a paraboloid) was on average parallel to the bottom. The tank was partially filled with water to an average depth of $H=19 \mathrm{~cm}$ and protected 
from air friction and evaporation by a false top separated from the free surface of the water by an air pocket of about $0.5 \mathrm{~cm}$ thick (while rotating). The convective plume was generated by introducing salty dyed water with a needle of inner diameter $d_{0}=1 / 16$ in. $(0.15875 \mathrm{~cm})$ sticking perpendicularly through the false top at $x=0$ and $y=5.8 \mathrm{~cm}$. The orifice of the needle was under water approximately $0.5 \mathrm{~cm}$ from the surface. Evolution of the flow was recorded by a digital still camera (and also by a video camera) attached to the centrifuge frame at a smaller radius. Two mirrors were attached to the tank by hinges in order to capture three simultaneous views (side and bottom views in addition to the front view) of the flow.

When all experimental devices had been assembled to the frame and the tank filled with the designed amount of water, the frame was slightly raised on a pivot located at the centre of the rotating platform and carefully balanced (within $0.1 \mathrm{~kg}$ ) by placing a counterbalance at the other end of the frame. Then the frame was firmly bolted to the platform. This procedure ensured the stability of rotation with accuracy $\Delta \Omega= \pm 0.001 \mathrm{~s}^{-1}$.

Adjustment and positioning of the optical parts were conducted in several steps. First, all sides of the tank were marked by coordinate lines. Then the suspension of the tank was adjusted to ensure that the $x$-axis was horizontal. After that, the camera was positioned in such a way that in the front view (view 'north') the coordinate lines $x=0$ on the 'southern' and 'northern' sides of the tank were aligned. Then, the side-view mirror (view 'west') was adjusted to ensure that the coordinate lines $y=0$ drawn on the 'eastern' and 'western' sides of the tank were also aligned. The bottom view mirror provided two useful choices: either a 'zenith' view (along the $z$-axis) if the mirror was placed at nearly $45^{\circ}$ to the bottom of the tank; or a 'polar' view (in the direction of $\boldsymbol{\Omega}$ ) if it was placed at a smaller angle, roughly $30^{\circ}$. We found the 'polar' view more helpful because the 'ink walls' were aligned with $\boldsymbol{\Omega}$. It is important to note that the adjustment of the mirrors had to be done with the tank filled with water as it has a different light refraction coefficient from air.

The syringe pump delivering the dyed salty water was located lower (smaller $\Phi$ ) than the needle's orifice in order to ensure positive pressure in the syringe and to avoid a siphon effect. We explored a range of volume flow rates $Q$ from $5 \mathrm{ml} \mathrm{h}^{-1}$ to $125 \mathrm{ml} \mathrm{h}^{-1}$, restricted by the syringe pump performance. The density of the plume $\rho_{1}$ ranged from $0.9987 \mathrm{~g} \mathrm{~cm}^{-3}$ to $1.1914 \mathrm{~g} \mathrm{~cm}^{-3}$. It was attained by adding saturated salt solution and some green food dye to the fresh tap water of density $\rho_{0}=0.9982 \mathrm{~g} \mathrm{~cm}^{-3}$ that was used to fill the tank. The density measurements were taken by a densitometer at $T=20^{\circ} \mathrm{C}$. Before each experiment, the temperature of the water in the tank and temperature of the plume water were adjusted to match the ambient air temperature in the laboratory, which ranged between 18 and $22^{\circ} \mathrm{C}$ depending on the weather. The temperature differences during the spin-up and the course of the experiment never exceeded $0.5^{\circ} \mathrm{C}$, which translates into an error in $\Delta \rho=\alpha_{w} \Delta T=1.0 \times 10^{-4} \mathrm{~g} \mathrm{~cm}^{-3}$, where $\alpha_{w}=2.07 \times 10^{-4}$ is the thermal expansion coefficient of water.

The crucial parameter characterizing the dynamics of the plume in our experiments is the buoyancy flux (with minus sign) delivered through the orifice of the needle (Morton et al. 1956)

$$
F_{0}=\frac{\rho_{1}-\rho_{0}}{\rho_{0}} g_{e} Q=g_{e}^{\prime} Q .
$$

We assume that it is switched on instantaneously as the dyed fluid appears at the orifice of the needle and remains constant afterwards. Note that (3.1) incorporates the 'effective' free-fall acceleration $g_{e}$ rather than $g$. As injection of the dyed water also 
introduces some momentum flux, it is necessary to estimate this effect. According to the Bernoulli equation

$$
w^{2}(z)=w_{0}^{2}+2 g_{e}^{\prime}\left(z_{0}-z\right)
$$

the vertical velocity $w$ within a uniform inviscid jet increases downward from the orifice $z_{0}$ owing to the density difference with the ambient fluid, where $w_{0}=4 Q /\left(\pi d_{0}^{2}\right)$ is the initial (average) velocity within the needle. For the distances exceeding

$$
\hat{z}_{b} \simeq \frac{w_{0}^{2}}{2 g_{e}^{\prime}},
$$

the buoyancy contribution will be dominant. For the most stringent combination of $Q=125 \mathrm{ml} \mathrm{h}^{-1}$ and $\Delta \rho=23 \times 10^{-4} \mathrm{~g} \mathrm{~cm}^{-3}$ that we used, $w_{0}=1.75 \mathrm{~cm} \mathrm{~s}^{-1}, g_{e}^{\prime}=$ $2.60 \mathrm{~cm} \mathrm{~s}^{-2}$, and $\hat{z}_{b}=0.56 \mathrm{~cm}$. Typically, however, $\hat{z}_{b}$ was less than $1 \mathrm{~mm}$; therefore the role of the momentum influx was insignificant. Thus, we essentially dealt with a point source of buoyancy.

The motions were generated by anomalies of salt concentration. The concentration of almost neutrally buoyant food dye was chosen to be $5 \%$ by volume of the injected fluid, as small as possible so as not to affect the dynamics, but still permit flow visualization. The density evolution equation for a small concentration of salt

$$
\rho_{t}+(\boldsymbol{v} \cdot \nabla) \rho=k_{S} \nabla^{2} \rho
$$

contains the diffusivity of salt $k_{S}$. Therefore, an analogue of the Prandtl number (called the Schmidt number) can be introduced $S c=v / k_{S}=O\left(10^{3}\right)$ which was large in our experiments.

\section{Development of the plume}

We chose the case of a plume developing from a point source in order to make a connection to some important previous laboratory and theoretical works. In a non-rotating environment, Turner (1962) demonstrated that a developing turbulent plume consists of a cap containing a vortex ring and a turbulent wake, all growing in a self-similar fashion with time. In particular, the distance $\hat{z}=z_{0}-z$ from the front to the origin grows with time as

$$
\hat{z} \simeq 1.8\left(F_{0} t^{3}\right)^{1 / 4}
$$

When the rotational axis is collinear with the direction of acceleration due to gravity $\alpha=0$, Fernando, Chen \& Ayotte (1998) and Maxworthy \& Narimousa (1994) showed that the rotational effects become important after a normalized time $\Omega t_{C 1} \simeq 2.4$, whence the vertical descent rate of the plume is slowed. Based on the data presented in Fernando et al. (1998, figure 10), the plume descent can be approximated by

$$
\hat{z} \simeq 2.1\left(\frac{F_{0}}{\Omega^{3}}\right)^{1 / 4}(\Omega t)^{0.6} \quad\left(t>t_{C 1}\right) .
$$

The coefficient in (4.2) follows from the requirement that at $t=t_{C 1}$, (4.1) and (4.2) match each other. The lateral growth of the plume is arrested at a time $\Omega t_{C 2} \simeq 5.5$. Thereafter, the vertical descent continues and the plume evolves into a cylindrical shape while developing a cyclonic circulation in and around it, except near the plume front. Upon reaching the bottom, the plume deflects, propagates horizontally and becomes unstable, breaking up into anticyclonic eddies. 


\begin{tabular}{|c|c|c|c|c|}
\hline Experiment & $\begin{array}{c}\Delta \rho \\
\left(10^{-4} \mathrm{~g} \mathrm{~s}^{-3}\right)\end{array}$ & $\begin{array}{c}g_{e}^{\prime} \\
\left(\mathrm{cm} \mathrm{s}^{-2}\right)\end{array}$ & $\underset{(\mathrm{mlh})}{Q}$ & $\begin{array}{c}F_{0} \\
\left(10^{-3} \mathrm{~cm}^{4} \mathrm{~s}^{-3}\right)\end{array}$ \\
\hline$C 1$ & 5 & 0.57 & 20 & 3.2 \\
\hline$C 2$ & 353 & 39.92 & 124.5 & 1381 \\
\hline$G$ & 23 & 2.61 & $\begin{array}{l}5 \\
7.5 \\
10 \\
15 \\
20 \\
40 \\
80\end{array}$ & $\begin{array}{c}3.62 \\
5.43 \\
7.25 \\
10.9 \\
14.5 \\
29.0 \\
58.0\end{array}$ \\
\hline$F$ & 100 & 11.34 & $\begin{array}{r}5 \\
15 \\
30 \\
60 \\
120\end{array}$ & $\begin{array}{r}15.75 \\
47.25 \\
94.50 \\
189.0 \\
378.0\end{array}$ \\
\hline$E$ & 121 & 13.722 & $\begin{array}{r}5 \\
10 \\
15 \\
20 \\
60 \\
120\end{array}$ & $\begin{array}{c}19.06 \\
38.12 \\
57.18 \\
76.23 \\
228.7 \\
457.4\end{array}$ \\
\hline$H$ & 509 & 57.723 & $\begin{array}{r}10 \\
20 \\
40 \\
80 \\
120\end{array}$ & $\begin{array}{l}160.3 \\
320.7 \\
641.4 \\
1283 \\
1924\end{array}$ \\
\hline$K$ & 1932 & 219.1 & $\begin{array}{r}10 \\
40 \\
80 \\
120\end{array}$ & $\begin{array}{r}608.6 \\
2434.4 \\
4868.8 \\
7303.2\end{array}$ \\
\hline
\end{tabular}

TABLE 1. Parameters of the experiments reported in the paper. Common to all experiments were $\Omega=1.509 \mathrm{~s}^{-1}, g=980.3 \mathrm{~cm} \mathrm{~s}^{-2}, \alpha=30^{\circ}, g_{e}=1132 \mathrm{~cm} \mathrm{~s}^{-2}, \rho_{0}=0.9982 \mathrm{~g} \mathrm{~s}^{-3}$, the depth of water $H=19 \mathrm{~cm}$.

What will be the new features of the flow when $\alpha$ is finite? Will the plume follow the direction of acceleration due to gravity, axis of rotation, or neither? How will its axial symmetry be broken? These are just a few important questions which we pursued. To illustrate the plume development, we shall discuss in detail the two experiments $C 1$ and $C 2$ corresponding to low and high $F_{0}$, respectively, which are given separately in table 1.

In experiment $C 1$, the buoyancy flux was relatively low, $F_{0}=3.2 \times 10^{-3} \mathrm{~cm}^{4} \mathrm{~s}^{-3}$ $\left(\Delta \rho=5 \times 10^{-4} \mathrm{~g} \mathrm{~s}^{-3}, Q=20 \mathrm{ml} \mathrm{h}^{-1}, g_{e}^{\prime}=0.57 \mathrm{~cm} \mathrm{~s}^{-2}\right)$, and the flow was in a weakly turbulent regime (in a sense that it was very chaotic, but lacked the apparent threedimensional turbulent billowing). The development of the dyed plume is depicted in figure 3 at three consecutive times, $t=55,476$ and $1592 \mathrm{~s}$, from the start of the plume, which was preceded by approximately one hour of spin-up.

Each photograph captures three views: view 'north' (top left), a mirror image of view 'west' (top right), and a mirror image of 'polar' view (bottom left). Since a mirror inverts the front and back and it is difficult to apprehend the depth dimension on 
the photograph, the mirror image of the view 'west' can be regarded as an equivalent view 'east'. Similarly, the mirror image of a 'polar' view can be regarded as a view in the direction opposite to $\boldsymbol{\Omega}$. In this way, we can keep thinking that all views depict a right-handed coordinate system. To eliminate confusion, axes $x, y$ and $z$ are shown on the photograph. Two tilted lines in the view 'west' represent the direction of the rotation axis.

The first snapshot at $t=55 \mathrm{~s}$ (figure $3 a$ ) shows the break-up of the inflowing dyed fluid into a number of blobs, which have a tadpole appearance. They propagate neither down nor along the axis of rotation, but mainly in the intermediate direction and also shift 'eastward'. Leading the group is the blob we designate as blob 1, eventually it will be the first blob to reach the bottom of the tank. The blobs leave behind a well-defined left-handed spiral-shaped tail. In the view 'west' (top right), it is seen that the spirals tend to be roughly aligned along the axis of rotation. The departure of the spirals from the perfect alignment is consistent with the result of Hide, Ibbetson \& Lighthill (1968) that the motion of a particle perpendicular to the axis of rotation produces a deflected Taylor column with the angle of deflection proportional to the Rossby number.

The second snapshot (figure $3 b$ ) is taken at $t=476 \mathrm{~s}$, when blob 1 has just reached the bottom. In the lower part of the plume, we can see a number of other distinct blobs: we designate as blob 2 and blob 3, those reaching the bottom second and third. In the upper part of the plume, the instability causing the break-up of the incoming jet also produces a diffuse cloud of more diluted dye. This fluid has a much smaller density difference with the surrounding water; therefore the cloud remains suspended for a much longer time. In the view 'west', this diffuse cloud clearly exhibits a tilted structure aligned along the axis of rotation: to the 'north' and 'south', the plume appears to be limited by two tilted lines parallel to $\boldsymbol{\Omega}$.

The third (last) snapshot at $t=1592 \mathrm{~s}$ depicts the stage when much of the dyed water has reached the bottom and is localized in the bottom Ekman layer. Morediluted dyed water is still suspended in the interior of the tank forming clearly defined tilted Taylor 'ink walls', seen especially well in the views 'north' and 'west'. At the same time, the 'polar' view confirms a depth-correlated character of the flow: looking through the whole water column along the $\boldsymbol{\Omega}$-direction, we see the eddies, which are associated with the Taylor 'ink walls', very clearly. If we looked through the water in a somewhat different direction, say strictly vertically, the eddies would be blurred due to overlapping.

Note that on the 'northern' side (in the view 'west'), the plume appears to be restricted by the tilted line. The two tilted lines are drawn on the 'eastern' side of the tank: one passes through the lower 'southern' corner, the other through the upper 'northern' corner. Between them is a region (having the shape of a parallelogram as seen in the view 'west') where the Taylor columns have a constant length $H / \cos \alpha$. Within this region, approximately geostrophic motions of fluid are possible. In the regions just outside, triangular in shape, fluid motions would result in finite stretching of the absolute vorticity tubes violating geostrophic approximation. Therefore, the tilted lines mark the 'impermeable' boundaries for the approximately geostrophic motions within the tank. The spread of the dyed fluid inside the region between the tilted lines happens much more rapidly, owing to eddies having a columnar depth-coherent structure, than its penetration outside, which is accomplished by the baroclinic effect and friction in the bottom Ekman layer.

Development of the plume in experiment $C 2$ with large $F_{0}=1.381 \mathrm{~cm}^{4} \mathrm{~s}^{-3}$ $\left(\Delta \rho=353 \times 10^{-4} \mathrm{~g} \mathrm{~s}^{-3}, Q=124.5 \mathrm{ml} \mathrm{h}^{-1}, g_{e}^{\prime}=39.92 \mathrm{~cm} \mathrm{~s}^{-2}\right)$ is illustrated in figure 4 

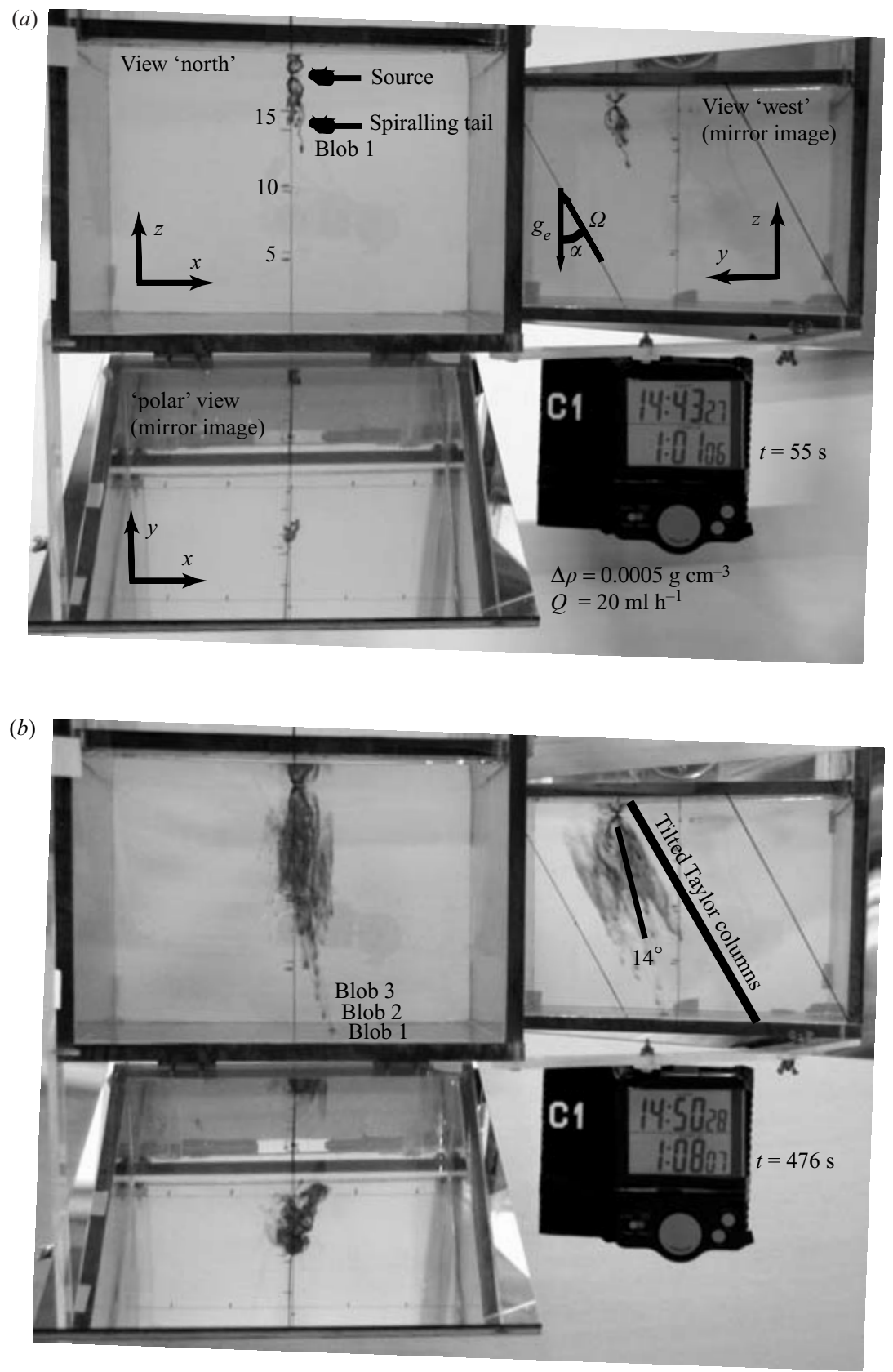

FIGURE $3(a, b)$. For caption see facing page.

also at three moments $t=2,39$ and $123 \mathrm{~s}$ from the start of the plume. Similar to experiment $C 1$, three stages of the flow are captured: initial instability of the entering jet, the plume reaching the bottom, and further lateral spread of the plume. Since the buoyancy flux parameter $F_{0}$, and hence typical velocities, are much larger in $C 2$ than in $C 1$, the plume development happens much faster. Also the flow appears to be highly turbulent: the entering jet rapidly disintegrates into turbulent billows 


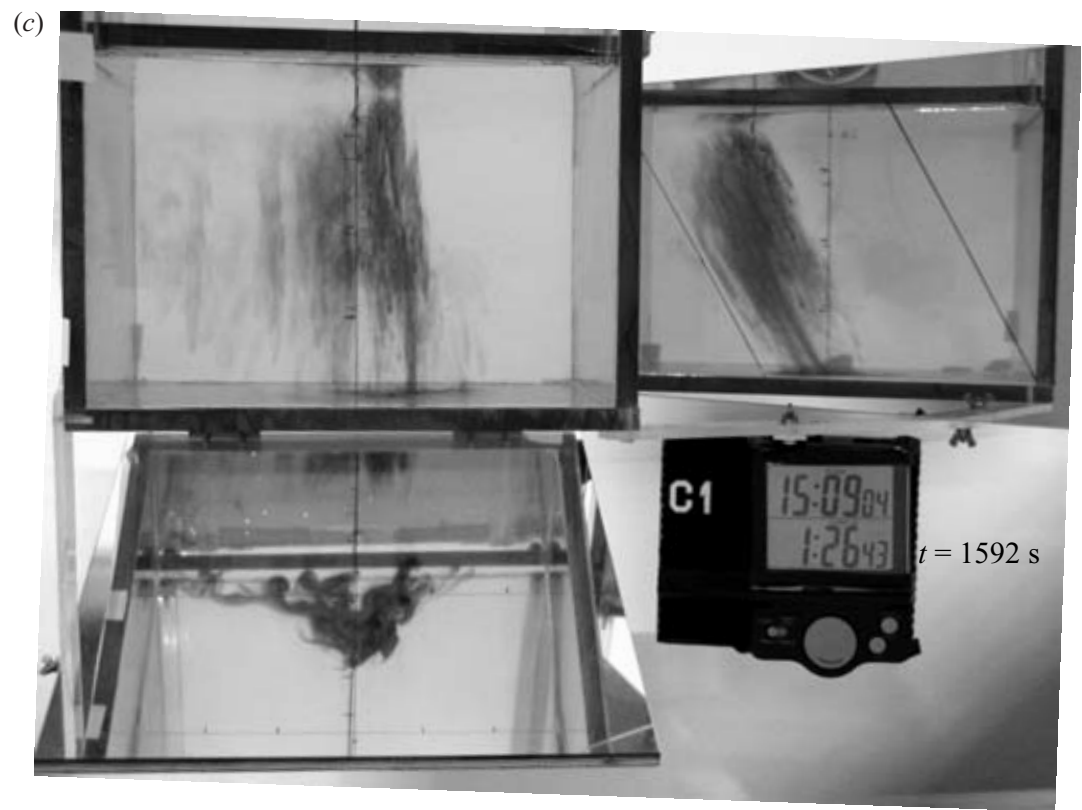

FiguRE 3. The development of the dyed plume in experiment $C 1$ with low $F_{0}$ at three consecutive times $(a) t=55 \mathrm{~s},(b) t=476 \mathrm{~s},(c) t=1592 \mathrm{~s}$ from the start of the plume. The 'hanging curtains' or Taylor 'ink walls' are visible in the view 'west', they are aligned with the axis of rotation which is marked by the two tilted lines. The scale in $(a)$ is in $\mathrm{cm}$. The angle $\theta_{y}=14^{\circ}$ according to (5.12) is marked in $(b)$.

(rather than blobs as in $C 1$ ) and a set of more diffuse tilted columns (figure $4 b$ ). Formation of the tilted structure appears through the similar mechanism: dilution of the incoming fluid by turbulent mixing followed by stretching along the direction of rotation with simultaneous shearing by horizontal motions. Upon the plume's impact with the bottom, two pairs of vortices are often generated travelling in opposite directions (figure $4 c$ ). These vortices have a small Rossby number; therefore they are accompanied by the tilted Taylor columns spanning the whole depth of the fluid.

To obtain a quantitative description of the plume development, we conducted several series of experiments $G, F, E, H$ and $K$ (see table 1). Within each series, the density anomaly $\Delta \rho$ was fixed, while the volume flow rate $Q$ was changed. Each experiment was usually repeated about three times in order to accumulate better statistics. The buoyancy flux parameter $F_{0}$ covered in these experiments spanned more than three orders of magnitude. Accordingly, the dynamics of the flow within the plume ranged from weakly (for small $F_{0}$ ) to highly turbulent (for large $F_{0}$ ).

One of the important quantities describing the evolution of the plume is the time $T_{B}$ it takes for the front of the plume to reach the bottom of the tank (to travel the distance $\hat{z}=z_{0}=18.5 \mathrm{~cm}$ ). Based on nearly a hundred experiments, in figure 5 we plotted $T_{B}$ as a function of the buoyancy flux parameter $F_{0}$. For large $F_{0}$, when the plume development is rapid and the rotation of the fluid is insignificant, scaling (Morton et al. 1956) suggests that $T_{B}$ should depend on the time scale $X=z_{0}^{4 / 3} F_{0}^{-1 / 3}$ constructed from the distance to the source and the buoyancy flux $(\log X$ is used as the abscissa in figure 5). The asymptotic scaling for a starting plume in an infinite non-rotating fluid according to Turner (1962, equation 13 , with $6 \alpha / 5=0.11$ and 

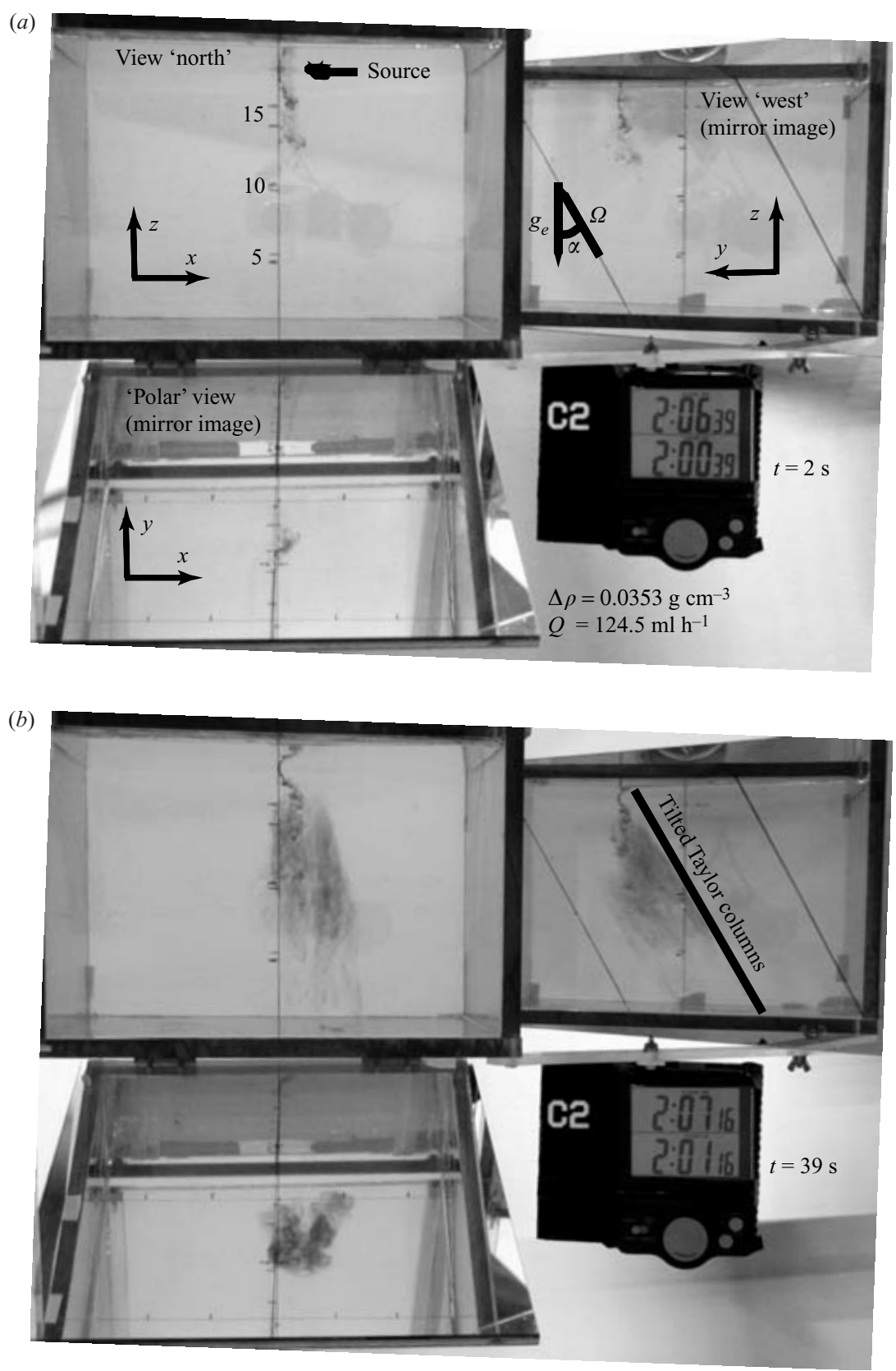

FIGURE $4(a, b)$. For caption see facing page.

$\left.c_{1}=0.38\right)$ or in other words the inversion of (4.1)

$$
T_{B} \simeq 0.46 z_{0}^{4 / 3} F_{0}^{-1 / 3}=0.46 X,
$$

is shown by the solid line for comparison. Similarly, substituting $T_{B}$ and $z_{0}$ into (4.2) and inverting it, we obtain the estimate (dashed line) based on the experimental 


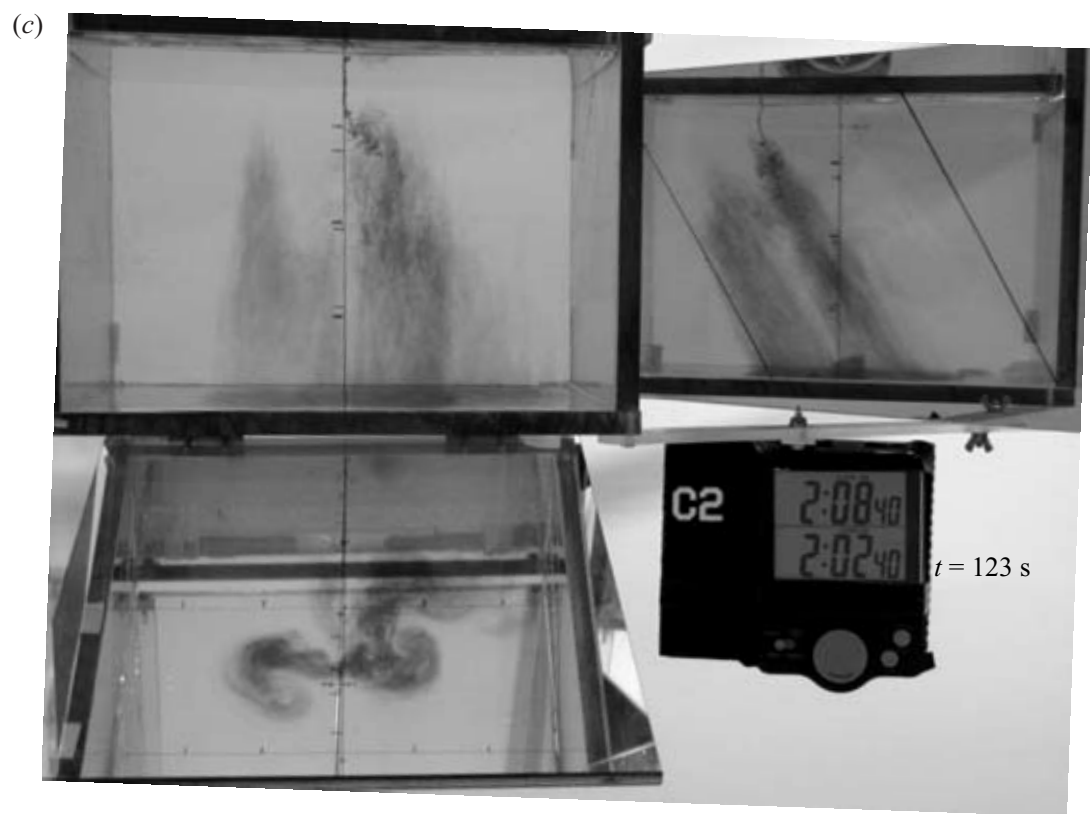

Figure 4. The development of the dyed plume in experiment $C 2$ with high $F_{0}$ at three consecutive times: (a) $t=2 \mathrm{~s},(b) t=39 \mathrm{~s},(c) t=123 \mathrm{~s}$ from the start of the plume. The scale in (a) is in $\mathrm{cm}$.

results of Fernando et al. (1998)

$$
T_{B} \simeq 0.30 \Omega^{-1}(X \Omega)^{1.25}
$$

which should be valid when rotation dominates or $T_{B}$ exceeds $2.4 \Omega^{-1}$.

We see that, in the tilted case, our experimental data fall somewhat closer to the non-rotating scaling (4.3) rather than to (4.4) over a broad range of $T_{B}$. Probably the mechanism suggested by Fernando et al. (1998) that the vertical descent rate of the plume is reduced due to the upward suction of plume fluid to supply the entrainment demand' does not operate as efficiently in the tilted case because of the drift of the plume off the axis of rotation. We remind the reader that the coefficient in Turner's theoretical formula is based on the assumption that a starting plume can be approximated as a combination of an advancing vortex ring located in the plume's cap and a steady wake behind the cap. A similar result, that the rotation only weakly affects the vertical propagation in the case of turbulent thermals (generated by an instantaneous point source) was found experimentally by Helfrich (1994).

However, for very small $F_{0}$, we find that the plume development slows down considerably. This happens when $T_{B}$ exceeds the spindown time scale

$$
T_{S}=\frac{1}{\Omega_{z}} \frac{H}{h_{E}}, \quad h_{E}=\sqrt{\frac{v}{\Omega_{z}}},
$$

caused by friction in the bottom Ekman layer. Formula (4.5) incorporates only the vertical component $\Omega_{z}$, similar to the case of a large-scale quasi-geostrophic flow. A careful analysis of the boundary-layer approximation (following Greenspan 1990, $\S 1.6)$ confirms its validity also in our case of a finite aspect ratio. For $t \ll T_{S}$, the introduction of a buoyancy source produces an accelerating flow in the ambient fluid. For $t \gg T_{S}$, however, the flow approaches a quasi-steady state with the balance 


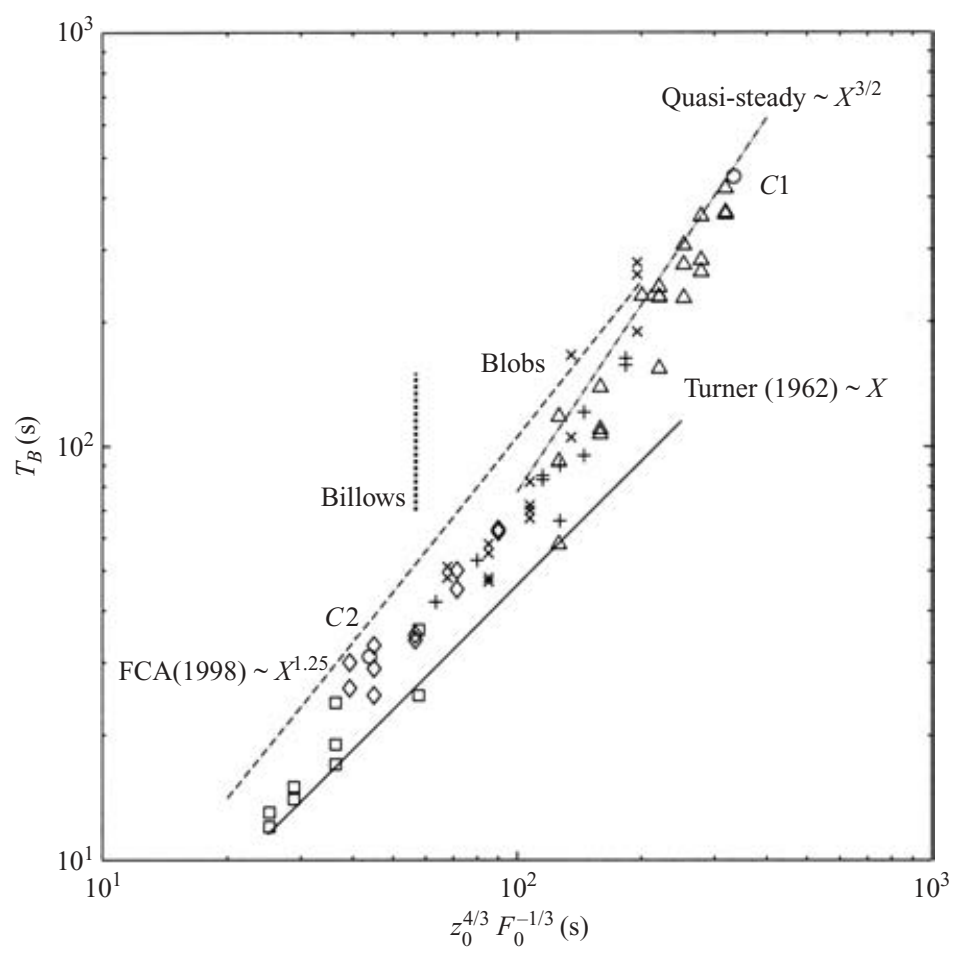

FIgURE 5. A plot of the time $T_{B}$ it takes the dyed fluid to reach the bottom (to travel the distance $z_{0}=18.5 \mathrm{~cm}$ ) versus the time scale $X$ dependent on the buoyancy flux parameter $F_{0}$. The asymptotic scaling for the starting plume in an infinite non-rotating fluid (4.3) according to Turner (1962) is shown by a solid line; the scaling for the rotating convection for $\alpha=0^{\circ}$ (4.4) according to Fernando et al. (FCA) (1998) is shown by a dashed line; the scaling for the quasi-steady regime (4.7) is shown by dash-dotted line. The approximate transition from convection with billows to convection with blobs is marked by the vertical dotted line. $\bigcirc, C 1$; $\bigcirc C 2 ; \triangle, G ; \times, F ;+, E ; \diamond, H ; \square, K$. See table 1 for values of $\Delta \rho$.

between the buoyancy forcing and dissipation in the Ekman boundary layer. The typical horizontal geostrophic velocities and vertical velocity, induced by the suction in the Ekman layer, depend on a pressure anomaly or a cumulative amount of the suspended mass anomaly which is proportional to $F_{0} t$. Therefore,

$$
w \simeq \frac{T_{S}}{H^{3}} F_{0} t,
$$

where $T_{S}$ (rather than $\Omega^{-1}$ ) and $H$ are used as the appropriate time and length scales. Since $w \simeq z_{0} / T_{B} \simeq H / T_{B}$ and $t \simeq T_{B}$ (4.6) can be rearranged to produce

$$
T_{B} \simeq\left(\frac{H^{4}}{T_{S} F_{0}}\right)^{1 / 2}=T_{S}^{-1 / 2} X^{3 / 2},
$$

with $X$ being the same time scale as in (4.3). The dashed-dotted line corresponding to (4.7) is also shown in figure $5, T_{S}=166 \mathrm{~s}$ in all our experiments. We can see that the data match it quite well for small $F_{0}$. The importance of the bottom Ekman-layer friction on the dye dispersion from a point source was also shown for the standard rotating tank experiments by Zatsepin (2001). 


\section{Blobs and drift of the plume}

We observed that in experiments without vigorous turbulent mixing, the dyed fluid forms conspicuous coherent blobs. Although such blobs are not directly related to the oceanic highly turbulent convection case, nonetheless, we will briefly discuss them since it will illuminate certain aspects of a larger-scale plume dynamics such as why the plume develops in the direction between the acceleration due to gravity and rotation vectors and shifts 'eastward'.

Blobs formed in all experiments with relatively small density anomaly $\Delta \rho$, hence $F_{0},(C 1, G, F, E, H)$ and did not in experiments with large $\Delta \rho, F_{0}(C 2, K)$. In the series $H$, we observed a gradual transition from turbulent billows to blobs as the flow rate was reduced (so was $F_{0}$ ). This transition is marked by the dotted vertical line in figure 5. By looking carefully, we can distinguish two ways of blob formation. First, blobs formed near the source owing to instability and wriggling motion of the incoming jet (figure $3 a$ ). The dynamics of this process is poorly understood, but probably is somewhat similar to the instability of a drop of milk in a cup of coffee (Mollo-Christensen 1972; Meng et al. 1998). Secondly, as the dyed fluid channelled along developing tilted Taylor 'ink walls' (or better to say 'tilted curtains') and accumulated at the lower end of the curtains (figure $3 b$ ), a blob formed which then descended at a somewhat steeper angle.

In experiment $C 1$, we traced the vertical propagation of the three individual blobs that were first to reach the bottom. The typical vertical velocities were rapidly decreasing from about $0.08 \mathrm{~cm} \mathrm{~s}^{-1}$ near the needle's orifice to $0.01-0.02 \mathrm{~cm} \mathrm{~s}^{-1}$ in the lower part of the tank, probably due to dilution of the density anomaly at the core. It is notable that most of the blobs had similar sizes; their shape could be approximated by an ellipsoid with the semi-axes $(a, a, c)$, with the major semi-axis $c$ aligned along $\boldsymbol{\Omega}$. The minor semi-axis $a$ (or half width) varied only within a narrow range between 0.13 and $0.16 \mathrm{~cm}$ from blob to blob, being on average $0.15 \mathrm{~cm}$ for blob 1 , and it barely changed along the trajectory of each blob. On the other hand, the longer axis $c$ (half length) had a larger dispersion, and was usually increasing as the blob descended; for example, blob 1 elongated from $c=0.28 \mathrm{~cm}$ at $z=15 \mathrm{~cm}$ to $c=0.40 \mathrm{~cm}$ at $z=5 \mathrm{~cm}$. Occasionally, some blobs suffered a transformation of their shape from an ellipsoid to a horseshoe, underwent an instability, and then rapidly diffused.

Based on the typical half-width of blob 1 in experiment $C 1 a=0.15 \mathrm{~cm}$, its vertical velocity $w=0.04 \mathrm{~cm} \mathrm{~s}^{-1}$, and the viscosity of water $v=0.01 \mathrm{~cm}^{2} \mathrm{~s}^{-1}$, we can estimate a typical Reynolds number $R e=a w / v=0.6$ and Rossby number $R o=w /(2 \Omega a)=0.09$. It is also convenient to speak in terms of the Taylor number $\mathscr{T}=\Omega a^{2} / v=3.4$, which is an inverse of the Ekman number $E=\mathscr{T}^{-1}=h_{E}^{2} / a^{2}=0.29$, where the Ekman thickness $h_{E}=\sqrt{v / \Omega}=0.081 \mathrm{~cm}$. Thus, we see that on the blob length scale, the frictional forces are significant and rotational effects are dominant.

As has already been mentioned, the most advanced blobs tend neither to follow the gravitational acceleration vector nor the rotation axis but propagate in the intermediate direction and also shift 'eastward' (figure $3 b$ ). This obviously has a bearing on the way the plume develops. To understand why the blobs propagate in a way they do, it is illuminating to consider them first as solid ellipsoids with the major semi-axes $(a, a, c)$ as mentioned above. In a steady motion (which is approximately true for the blobs), the balance of forces acting on an ellipsoid,

$$
\boldsymbol{F}_{C}+\boldsymbol{F}_{D}+\boldsymbol{F}_{L}+\boldsymbol{F}_{B}=0
$$

includes, respectively, the Coriolis force, the hydrodynamic drag and lift due to the dynamic pressure and viscous stresses at its surface, and the buoyancy force 
resulting from the hydrostatic pressure field. The most difficult part is to calculate the hydrodynamic forces because, when a particle moves in a rapidly rotating fluid at small Rossby number, they have a very peculiar character due to formation of a Taylor column around the particle which was first predicted theoretically by Proudman (1916) and Taylor (1916) and then confirmed experimentally by Taylor (1922). Bush, Stone \& Tanzosh (1994) reviewed our theoretical knowledge on a particle motion in a rotating fluid in various regimes. We would like especially to note the work of Stewartson (1953) who, using a Laplace transform, solved a linear inviscid problem of an ellipsoid impulsively started from rest in a rotating fluid. In the limit of large time, he calculated the pressure distribution on the surface of an ellipsoid and hence found the drag and lift on a uniformly moving ellipsoid due to generation of inertial waves and development of a Taylor column.

Stewartson (1953) found that in a motion along the axis of rotation with velocity $V_{h}$, an ellipsoid experiences a drag

$$
F_{D h}=-\frac{16}{3} \rho_{0} a^{3} \Omega V_{h},
$$

while moving perpendicular to the axis of rotation with, for example, velocity $V_{r}$ it experiences both the drag

$$
F_{D r}=-\frac{4}{3} \pi a^{2} c \rho_{0} \frac{4 \pi a c}{16 a^{2}+\pi^{2} c^{2}} 2 \Omega V_{r},
$$

and lift

$$
F_{L \lambda}=\frac{4}{3} \pi a^{2} c \rho_{0} \frac{\pi^{2} c^{2}}{16 a^{2}+\pi^{2} c^{2}} 2 \Omega V_{r},
$$

acting to the left if viewed from the end of $\boldsymbol{\Omega}$. Here, we use the non-tilted system of coordinates $(r, \lambda, h)$ to indicate directions. For small density difference between the particle and fluid, $\Delta \rho \ll \rho_{0}$, the Coriolis force

$$
F_{C \lambda}=-\frac{4}{3} \pi a^{2} c\left(\rho_{0}+\Delta \rho\right) 2 \Omega V_{r},
$$

acting to the right, but stronger than the lift force, can be joined to a single matrix equation relating the velocity of an ellipsoid and the sum of drag, lift and Coriolis forces acting on it (since they are linearly dependent on velocity)

$$
\left(\begin{array}{c}
F_{r} \\
F_{\lambda} \\
F_{h}
\end{array}\right)=-\frac{16}{3} \rho \Omega a^{3}\left[\begin{array}{ccc}
\frac{2 \pi^{2}}{16(a / c)^{2}+\pi^{2}} & -\frac{8 \pi a / c}{16(a / c)^{2}+\pi^{2}} & 0 \\
\frac{8 \pi a / c}{16(a / c)^{2}+\pi^{2}} & \frac{2 \pi^{2}}{16(a / c)^{2}+\pi^{2}} & 0 \\
0 & 0 & 1
\end{array}\right]\left(\begin{array}{c}
V_{r} \\
V_{\lambda} \\
V_{h}
\end{array}\right) .
$$

In order to obtain the velocity of an ellipsoid resulting from applied buoyancy force

$$
\boldsymbol{F}_{B}=\frac{4}{3} \pi a^{2} c \Delta \rho \boldsymbol{g}_{e},
$$

we invert this matrix and use the balance (5.1) to obtain

$$
\left(\begin{array}{l}
V_{r} \\
V_{\lambda} \\
V_{h}
\end{array}\right)=\frac{\pi}{4 \Omega} \frac{\Delta \rho}{\rho} \frac{c}{a}\left[\begin{array}{ccc}
\frac{1}{2} & \frac{2}{\pi} \frac{a}{c} & 0 \\
-\frac{2}{\pi} \frac{a}{c} & \frac{1}{2} & 0 \\
0 & 0 & 1
\end{array}\right]\left(\begin{array}{l}
g_{e r} \\
g_{e \lambda} \\
g_{e h}
\end{array}\right)
$$


In our case, the 'effective' gravitational acceleration vector projected onto $(r, \lambda, h)$ has the following components $\left(g_{e r}, g_{e \lambda}, g_{e h}\right)=\left(\Omega^{2} R, 0,-g\right)=\left(g_{e} \sin \alpha, 0,-g_{e} \cos \alpha\right)$. Using the rotation transformation

$$
V_{x}=-V_{\lambda}, \quad V_{y}=\cos \alpha V_{r}+\sin \alpha V_{h}, \quad V_{z}=-\sin \alpha V_{r}+\cos \alpha V_{h},
$$

we find velocity components in the coordinate frame $(x, y, z)$

$$
\left(\begin{array}{l}
V_{x} \\
V_{y} \\
V_{z}
\end{array}\right)=\frac{\pi}{4 \Omega} \frac{\Delta \rho}{\rho} g_{e}\left(\begin{array}{c}
(2 / \pi) \sin \alpha \\
-(c / 4 a) \sin 2 \alpha \\
-(c / 4 a)(3+\cos 2 \alpha)
\end{array}\right) .
$$

Thus, as an ellipsoid sinks $\left(V_{z}<0\right)$, it drifts 'southward' $V_{y}<0$ and 'eastward' $V_{x}>0$ along a straight trajectory inclined to the vertical through angles $\theta_{x}$ and $\theta_{y}$ determined by

$$
\begin{gathered}
\tan \theta_{x}=\frac{V_{x}}{V_{z}}=-\frac{8}{\pi} \frac{a}{c} \frac{\sin \alpha}{3+\cos 2 \alpha}, \\
\tan \theta_{y}=\frac{V_{y}}{V_{z}}=\frac{\sin 2 \alpha}{3+\cos 2 \alpha} .
\end{gathered}
$$

Note that the 'southward' drift angle $\theta_{y}$ is independent of $a / c$ while the 'eastward' drift angle $\theta_{x}$ decreases as the ellipsoid is elongated $(a / c<1)$ along $\boldsymbol{\Omega}$.

For illustration of the above formulae, we shall focus again on blob 1. Its trajectory in the $(x, z)$ - and $(y, z)$-planes is plotted in figure 6 by open circles based on the data from consecutive photographs. Two views are presented simultaneously: the left-hand side of the plot shows the $y$-coordinate, while the right-hand side shows the $x$-coordinate. The source of the plume at $x, y=0$ and $z=18.5$ is shown by a star. (In figure 6 the origin of the $y$-axis is assumed to coincide with the needle's orifice). For $\alpha=30^{\circ}$ (5.11), (5.12) predict the drift angles $\theta_{y}=14^{\circ}$ (independent of $a / c$ ) and $\theta_{x}=-10^{\circ}$ ( for $a / c=0.5$ as observed for blob 1 near the origin). The corresponding theoretical trajectories are shown by dotted lines, in excellent agreement with observations.

Both nonlinearity and viscosity relax the Taylor-Proudman constraint; therefore, the Taylor column has a finite extent (a viscous theory suggests $O(a \mathscr{T})$, approximately $0.5 \mathrm{~cm}$ for blob 1). Loper (2001) pointed out important differences between the dynamics of solid particles and buoyant fluid parcels (such as our blobs) in a rotating fluid. Based on a linear viscous theory valid for small Rossby number and high Taylor numbers (which is applicable to our blobs at least marginally), he calculated the flow inside and outside a buoyant fluid parcel, and its motion in a general case of misaligned $\boldsymbol{\Omega}$ and $\boldsymbol{g}_{e}$. His result for the trajectory of a spherical fluid parcel is identical with the prediction based on Stewartson's (1953) formula, which completely neglects viscosity, for a solid spherical particle. Unfortunately, in comparing with Stewartson's results, Loper omitted the Coriolis force in his equation (8.8), leading to an inaccurate comparison. In fact, the force-velocity relations for a spherically symmetric fluid parcel (Loper's equation (8.9)) and a rigid sphere (our equation (5.8) with $a=c$ ) are identical. The applicability of Stewartson's formula for solid spheres was tested experimentally using a similar setup to that reported here; the preliminary results can be found in Riemenschneider (2002). Essentially, hydrodynamic reaction of the fluid on a moving particle or a parcel is determined by the (approximately linear) flow around a truncated Taylor column, which is common in both Stewartson's and Loper's work. However, the establishment of the Taylor column is limited by 


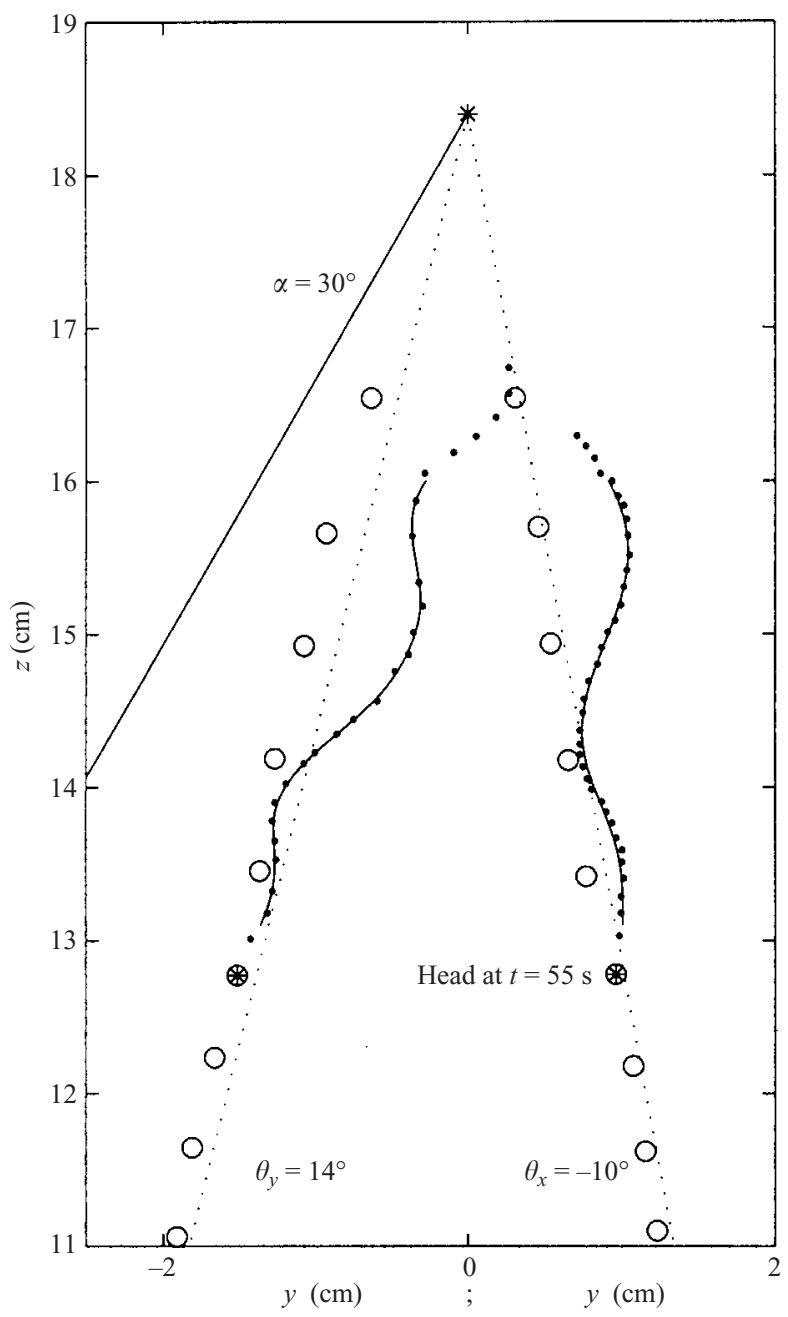

FIgURE 6. Analysis of the trajectory and tail of blob 1 in experiment $C 1$. Two views are superimposed on the same plot: the data on the left correspond to the $y$-coordinate, the data on the right to the $x$-coordinate. The trajectory of the blob is straight. It is indicated by open circles corresponding to different moments separated by approximately $4 \pi \Omega^{-1}$, or roughly $8 \mathrm{~s}$. The tail, which was digitized from the photograph in figure $3(a)$ and shown by small dots connected by a spline, forms a left-handed spiral. The head of the blob at $t=55 \mathrm{~s}$ as in figure $3(a)$ is shown with the filled circle. The star at the top indicates the needle's orifice. The solid line (on the left) emanating from the star is the axis of rotation having $\alpha=30^{\circ}$ with the vertical. The dotted line (on the left) inclined by $\theta_{y}=14^{\circ}$ is a prediction of a linear theory for the 'southward' drift according to (5.12). The dotted line (on the right) inclined by $\theta_{x}=-10^{\circ}$ is a prediction of a linear theory for the 'eastward' drift of an ellipsoid with $c / a=2$ according to $(5.11)$.

the time-dependent effects in the case of Stewartson and by weak viscous effects in the case of Loper. We also note that the propagation angles are independent of the buoyancy anomaly and, therefore, should not change as the blob descends and slows down due to the dilution of its core.

In our case, the presence of the truncated Taylor column is manifested by the spiralling tail that the blob leaves in its wake. The coordinates of the tail of blob1, 
which were digitized from the photograph in figure $3(a)$, are also plotted in figure 6 by small dots connected by a smooth spline. The filled circle indicates the position of the head of the blob at time $t=55 \mathrm{~s}$ corresponding to the photograph in figure $3(a)$. Because the trajectory of the blob is straight rather than oscillatory, it follows that spiralling of the tail is caused by circulation in the wake of the blob. A plot (not shown) of the tail in the plane $(x, y)$ parametrically as a function of $z$ would reveal that the spiral is left-handed. The same result holds for other blobs as well. As the fluid flows around the blob, it first tends to diverge causing anticyclonic circulation and high pressure in front of the blob and then tends to converge causing cyclonic circulation and low pressure in the blob's wake. This mechanism essentially determines the increased hydrodynamic drag in rotating fluids. As the dye is shed from the blob, it is entrained by the cyclonic circulation (counterclockwise if viewed from above) and thus acts as a source propagating along a left-handed spiral.

We have to make a few comments regarding the applicability of (5.8). It predicts the drift of a particle (or of a fluid parcel as shown by Loper 2001) relative to the stagnant (in a rotating reference frame) surrounding fluid. As the plume develops, it generates large-scale circulation spanning the whole depth of the tank, which will affect the apparent trajectory of a blob. However, for the first blobs moving outside of the developing plume, the effect of the plume is small.

Thus, the analysis of propagation of individual blobs helps us to understand why the plume (if we think of it as an ensemble of blobs) in the beginning tends to develop in the direction between the acceleration due to gravity and rotation vectors and shifts 'eastward'. Since the propagation angles are independent of the buoyancy anomaly, even for very weak sources of buoyancy $F_{0}$, the convection will never be restricted to the axis of rotation; there always will be off-axis motions. This destroys possible symmetry with respect to the axis of rotation and causes much more efficient horizontal spread of the convective plume compared to the case when $\boldsymbol{\Omega}$ and $\boldsymbol{g}_{e}$ are aligned. Also we believe that the balance (5.8) including the buoyancy, Coriolis forces, and the hydrodynamic reaction induced by inertial waves is robust and generic, and should be applicable to any coherent features within the oceanic convecting plume.

Finally, it is interesting to make a connection with the work of Straneo et al. (2002) who applied the theory of slantwise convection to the oceanic case with the horizontal component of rotation. They considered a Lagrangian dynamics of a horizontally (zonally) infinite cylindrical parcel conserving the absolute angular momentum and found that, following a complicated motion, such a cylinder will eventually sink along the axis of rotation. If we consider an arbitrary ellipsoid with semi-axes $(a, b, c)$ aligned with $(\lambda, r, h)$ and $a \gg b, c$, as an approximation for a zonally oriented cylinder, then according to Stewartson (1953, equations (5.12), (6.24), (6.25)) the hydrodynamic reaction will incorporate the complete elliptic integrals of the first and the second kind, $K$ and $E$ of the modulus $\left(a^{2}-b^{2}\right)^{1 / 2} / a$. For $a / b \rightarrow \infty, K \rightarrow \infty, E \rightarrow 1$ and the formulae simplify. For the motion along the axis of rotation, the drag is $F_{D h}=-8 \rho_{0} a b^{2} \Omega V_{h} / 3$. For the motion perpendicular to $\boldsymbol{\Omega}$, the drag force $F_{D r}$ vanishes, while the lift force is $F_{L \lambda}=8 \pi \rho_{0} a b c \Omega V_{r} / 3$ and is exactly compensated by the Coriolis force for $\Delta \rho \ll \rho_{0}$, similarly for the motion in the direction $\lambda$. Thus, the matrix in (5.6) will have only one non-zero element, corresponding to the drag along the axis of rotation, and the steady motion under misaligned $\boldsymbol{g}_{e}$ will be impossible. Therefore, it is then necessary to consider relative acceleration of a parcel which will result in transient motions in agreement with Straneo et al. (2002). However, on horizontal scales comparable with the depth of fluid, such a cylindrical parcel will probably be unstable and disintegrate into much smaller parcels with $a \simeq b$, which then will approximately obey (5.10). 


\section{Tilted Taylor 'ink walls'}

The most arresting feature of the convection with misaligned $\boldsymbol{\Omega}$ and $\boldsymbol{g}_{e}$ is formation of tilted structures or tilted Taylor 'ink walls' which were observed in all our experiments. Their formation is accomplished by a process of dye spreading along the tilted absolute vorticity lines and simultaneous shearing by horizontal quasi-twodimensional flow. It is accurately described by Long (1954): 'If we suddenly plunge a foreign body of fluid into a system in solid cyclonic rotation, the vortex lines will be forced apart, and as a result the relative circulation on horizontal rings of fluid surrounding the foreign fluid will become negative. Initially, the resulting anticyclonic vortex will not have a balance of pressure-gradient force and Coriolis force, and the rings will contract. This will force the injected fluid to spread vertically until it reaches the bottom of the vessel and the free surface. The relative motion will now be two-dimensional, and the newly formed ink wall will be stretched out horizontally'.

The same mechanism also works when the intrusion has a slight buoyancy anomaly. In our experiments, we clearly see disintegration of the incoming fluid into denser faster sinking coherent features such as blobs or turbulent billows and a diffuse cloud of highly diluted dyed water, which can remain suspended for a long time and eventually form tilted 'ink walls'. With a continuous supply of dyed fluid there is a continuous source of turbulent energy. However, beyond a certain depth the three-dimensional turbulence in the billows becomes suppressed by the rotation when the Rossby number of the turbulent motion becomes small $v^{\prime} / 2 \Omega l \simeq 1$, where $v^{\prime}$ and $l$ are velocity and length scales of the dominant turbulent motions. According to Fernando et al. (1998) and Maxworthy \& Narimousa (1994), this happens roughly at a distance (see (4.2))

$$
\hat{z}_{C 2}=6.5\left(\frac{F_{0}}{\Omega^{3}}\right)^{1 / 4} .
$$

For example, in experiment $C 2, \hat{z}_{C 2}=5.2 \mathrm{~cm}$, accordingly we see that the turbulent billows decay beyond $\hat{z}_{C 2}$ and the tilted structures appear to originate around that depth (figure 4). The turbulent mixing along with a lack of axial symmetry greatly dilutes the dye of the incoming jet, reducing buoyancy anomalies relative to the ambient fluid. Again in experiment $C 2,1.34 \mathrm{~cm}^{3}$ was injected by $t=39 \mathrm{~s}$ (figure $4 b$ ) which is seen spread over much of the water column indicating a high dilution factor of about 300 .

Apart from the turbulent entrainment which causes flow convergence and drives cyclonic circulation around the plume, in our case of a finite $\alpha$, the two-dimensional horizontal motions are also generated by the tendency of the plume to shift off the axis of rotation. In particular, according to Loper (2001), the pressure anomaly around a blob decays only algebraically $P\left(h^{\prime}\right) \simeq\left(E\left|h^{\prime}\right|\right)^{-2 / 3}$ with axial distance $h^{\prime}$, and, therefore, a weak influence extends much farther than the scale $a \mathscr{T}$. Thus, descending blobs drifting off the axis, to some extent, drag along the tilted Taylor columns with highly diluted dye. That is why we see (figures 3 and 4 ) that the plume has very sharp tilted boundaries aligned with the axis of rotation. The off- $\Omega$-axis drift results in a more efficient overall spread of the dyed fluid, as compared to the axially symmetric case $\alpha=0$, when the spread is restricted by the axial symmetry. It also promotes the generation of the two-dimensional quasi-geostrophic turbulence which sharpens two-dimensional gradients of the tracer and thus plays an important role in producing the 'ink walls'.

Dynamical insight into formation of the tilted structures can be gained by considering the equation for the relative vorticity $\zeta$, which is obtained by taking 
the curl of (2.1) and using (2.2)

$$
\frac{\partial}{\partial t} \zeta+(\boldsymbol{v} \cdot \nabla) \zeta-(\zeta \cdot \nabla) \boldsymbol{v}-2(\boldsymbol{\Omega} \cdot \nabla) \boldsymbol{v}=\frac{\boldsymbol{g}_{e}}{\rho_{0}}(\hat{k} \times \nabla \rho)+v \nabla^{2} \zeta
$$

where the baroclinic term is written down in Boussinesq approximation $\left(\Delta \rho \ll \rho_{0}\right)$. The rotation dominates when the fourth term in (6.2) is much larger than the rest. This happens when the Rossby number of the flow is small,

$$
\epsilon=|\zeta| / \Omega=U /(\Omega L) \ll 1
$$

and the variation of the density field is weak

$$
\frac{\Delta \rho}{\rho_{0}} \frac{g_{e}}{L} / \frac{2 \Omega U}{H}=O(\epsilon) \ll 1,
$$

where $U$ is the typical velocity scale and $L$ is the typical horizontal scale, which can be as small as the vertical scale $H$. Carrying out a standard expansion of all variables in $\epsilon$, at the zeroth order we obtain the Taylor-Proudman constraint

$$
2(\boldsymbol{\Omega} \cdot \nabla) \boldsymbol{v}^{0}=0
$$

which in our case permits a solution satisfying the boundary conditions $w^{0}(0)=$ $w^{0}(H)=0$

$$
u^{0}=-\frac{\partial}{\partial y} \psi^{0}(x, y-z \tan \alpha, t), \quad v^{0}=\frac{\partial}{\partial x} \psi^{0}(x, y-z \tan \alpha, t), \quad w^{0}=0,
$$

where $\psi^{0}$ is the geostrophic streamfunction, which shows that the geostrophic motions are aligned along $\boldsymbol{\Omega}$ and tilted in the $(y, z)$-plane.

At the first order, we obtain

$$
\frac{\partial}{\partial t} \zeta^{0}+\left(\boldsymbol{v}^{0} \cdot \nabla\right) \zeta^{0}-\left(\zeta^{0} \cdot \nabla\right) \boldsymbol{v}^{0}-2(\boldsymbol{\Omega} \cdot \nabla) \boldsymbol{v}^{1}=\frac{\boldsymbol{g}_{e}}{\rho_{0}}\left(\hat{k} \times \nabla \rho^{1}\right),
$$

where we assumed that the expansion of the density field starts with the first-order term (corresponding to more diffuse suspended matter than the blobs or billows are) and the viscous term can be neglected except in the bottom boundary layer. Taking into account (6.6), the projections of (6.7) onto $(x, y, z)$ can be written in a simplified form

$$
\begin{aligned}
\tan \alpha \frac{\partial}{\partial y}\left(\frac{\partial}{\partial t} v^{0}+J\left(\psi^{0}, v^{0}\right)\right) & =2 \Omega \frac{\partial}{\partial h} u^{1}-\frac{g_{e}}{\rho_{o}} \frac{\partial}{\partial y} \rho^{1} \\
\tan \alpha \frac{\partial}{\partial y}\left(\frac{\partial}{\partial t} u^{0}+J\left(\psi^{0}, u^{0}\right)\right) & =2 \Omega \frac{\partial}{\partial h} v^{1}+\frac{g_{e}}{\rho_{o}} \frac{\partial}{\partial x} \rho^{1}, \\
\frac{\partial}{\partial t} \zeta^{0}+J\left(\psi^{0}, \zeta^{0}\right) & =2 \Omega \frac{\partial}{\partial h} w^{1},
\end{aligned}
$$

where

$$
J(a, b)=\frac{\partial b}{\partial x} \frac{\partial a}{\partial y}-\frac{\partial a}{\partial x} \frac{\partial b}{\partial y}
$$

is the Jacobian,

$$
\zeta^{0}=\left(\frac{\partial^{2}}{\partial x^{2}}+\frac{\partial^{2}}{\partial y^{2}}\right) \psi^{0}
$$


and

$$
\Omega \frac{\partial}{\partial h}=\Omega_{y} \frac{\partial}{\partial y}+\Omega_{z} \frac{\partial}{\partial z}
$$

is the gradient in the direction of $\boldsymbol{\Omega}$.

Similar to the standard case of aligned $\boldsymbol{\Omega}$ and $\boldsymbol{g}_{e}$ or in a shallow-water approximation $H \ll L$, the equation for the slow evolution of the geostrophic fields comes as the solvability condition of (6.10) which is the first-order equation in $h$ subject to two boundary conditions on $w^{1}$. In general, to close the problem, we have to take into account the density evolution equation (3.4) and the Ekman suction in the bottom boundary layer, since (6.8) and (6.9) do not contribute to $w^{1}$. Even without doing so, it is interesting to note an important distinction. In the standard case, if we assume no vertical stretching $(w \equiv 0)$ as in inviscid problem and no horizontal variations in density, the quasi-geostrophic evolution equation (6.10) reduces to a two-dimensional vorticity advection equation, permitting an exactly two-dimensional 'barotropic' motion (in general, even with an arbitrary stratification $\rho=\rho(z)$ ). There is no vertical shear, (6.8) and (6.9) are satisfied trivially. In particular, a circular cylindrical vortex (with streamlines being circles in the $(x, y)$-plane) is an exact steady solution of the full nonlinear equation. In the tilted case, an analogue of this barotropic solution still exists; however, the motion is two-dimensional only approximately, $u$ and $v$ fields have $O(\epsilon)$ shear since the left-hand sides of (6.8) and (6.9) no longer vanish. A tilted circular cylindrical vortex is a steady solution of the first-order quasigeostrophic equation (6.10) for $\epsilon \ll 1$, but not of the full nonlinear equation. This is due to the fact that a vortex line tilted with respect to a solid boundary cannot remain steady because of the interaction with its image. Specifically, the reflection at the boundary is equivalent to a kink (or a point with infinite curvature) in a vortex line, which causes a self induced motion (Batchelor 1967, chap. 7.1).

We also note that an almost steady cylindrical vortex has a circular cross-section in the $(x, y)$-plane as follows from (6.10) rather than in the plane perpendicular to $\boldsymbol{\Omega}$, which is due to the boundary conditions on $w$. Such a cylindrical vortex is of special interest because, if the density field has a cylindrical symmetry, it will remain approximately unchanged even in the presence of a small shear as in (6.8), (6.9) which is parallel to the main geostrophic flow. Finally, we note that although the above expansion is formally valid for small $\epsilon$, it is often found that qualitatively the quasi-geostrophic expansion works well even for $\epsilon \simeq 1$.

Now we return our attention to a geophysically relevant application: the ocean deep convection. A special issue of the Journal of Physical Oceanography (February 2002) contains a collection of papers presenting the results of the recent 'Labrador Sea Deep Convection Experiment'. Observations with the Lagrangian floats (Steffen \& D'Asaro 2002) as well as fixed mooring observations (Lilly \& Rhines 2002) suggest that the evolution of plumes often spans several days and that the associated Rossby number is small, about 0.2 . Also the deep convection is characterized by exceptional homogenization of water within the mixed layer, $\Delta \rho / \rho_{0} \simeq 10^{-6}$ over $1 \mathrm{~km}$ of depth and $\Delta \rho / \rho_{0} \simeq 4 \times 10^{-5}$ over $200 \mathrm{~km}$ horizontally (Pickart et al. 2002) implying that the vertical geostrophic shear is small. In fact with $2 \Omega=10^{-4} \mathrm{~s}^{-1}$ and $U=10 \mathrm{~cm} \mathrm{~s}^{-1}$, the ratio in (6.4) is about 0.2. Thus, conditions are favourable for the formation of tilted structures similar to the tilted Taylor 'ink wall' observed in our experiments. The role of the dye in the ocean is played by the temperature $T^{\prime}$ (or potential temperature $\theta^{\prime}$ ) and salinity $S^{\prime}$ anomalies, or even better by the so-called 'spice' $\tau$, which is the quantity orthogonal to the density in the temperature-salinity space according to the 

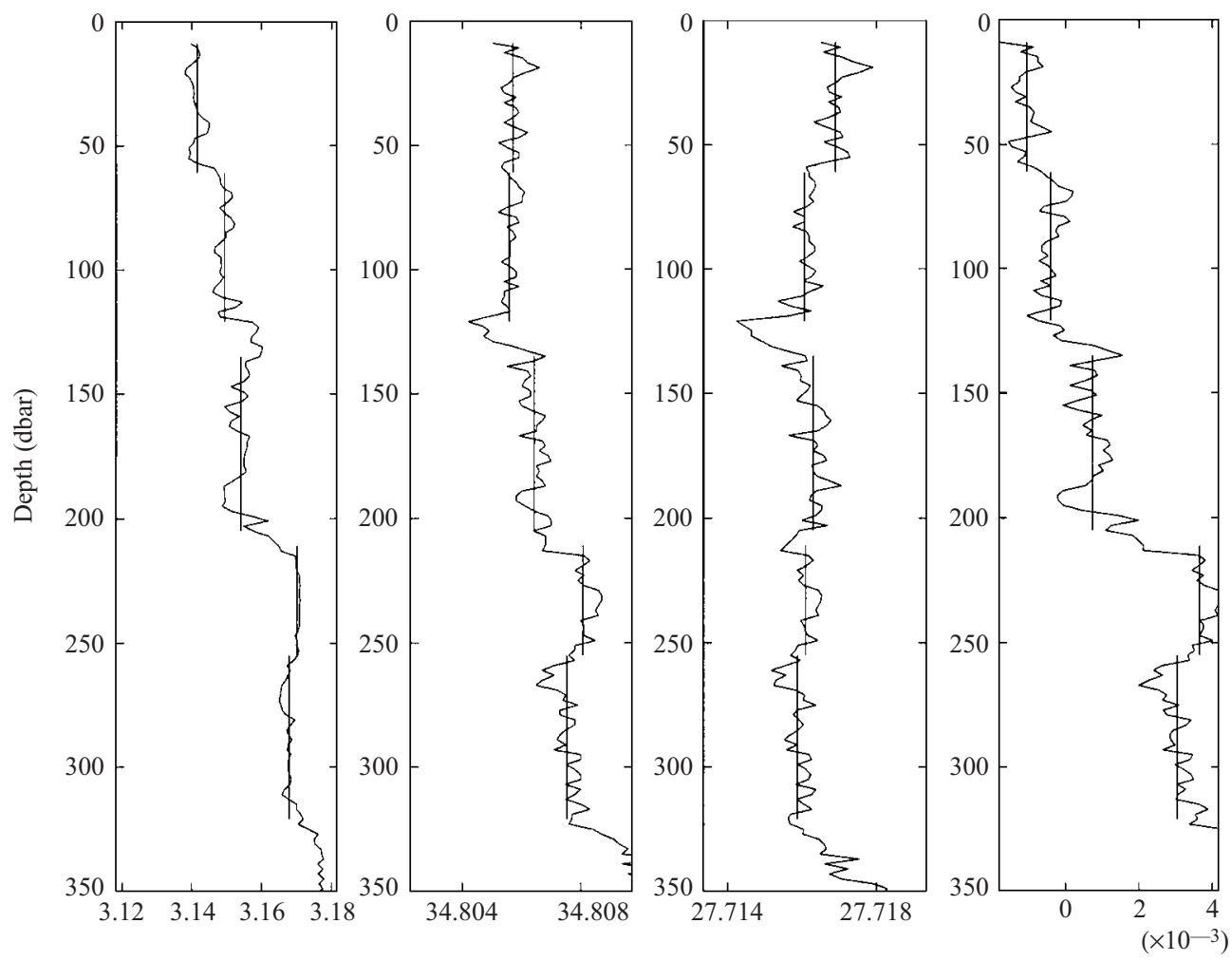

FiguRE 7. Vertical profiles of $(a)$ potential temperature $\theta,(b)$ salinity, $(c)$ potential density $\sigma_{0}$, and $(d)$ spice $\tau$ through an actively convecting layer. The vertical scale (pressure) is in dbar which corresponds to approximately $1 \mathrm{~m}$ of depth. Smaller scale homogenized sublayers extending 50-100 m (marked by vertical segments) are visible within the convective layer. Shown only is the upper portion of the mixed layer which extended to about $750 \mathrm{~m}$ at this location. The data were collected during the Deep Convection Experiment in February 1997 in the western Labrador Sea from R/V Knorr, Leg 147, Station 52 (provided by R. S. Pickart).

equation of state of sea water (Veronis 1972; Mamayev 1975; Munk 1981)

$$
\tau=-\left(\frac{\partial \sigma_{0}}{\partial \theta}\right)_{S} \theta^{\prime}+\left(\frac{\partial \sigma_{0}}{\partial S}\right)_{\theta} S^{\prime},
$$

where the potential density $\sigma_{0}$ and the potential temperature $\theta$ referenced to the atmospheric pressure are introduced to take into account the compressibility of sea water. The 'spice' acts as a passive tracer when the contributions of $\theta^{\prime}$ and $S^{\prime}$ to the density field are compensated. Thus in the ocean, as a CTD travels vertically and intersects the tilted 'ink walls', it should detect steps in temperature, salinity, density, and most pronounced in spice, which indeed have been observed in convectively mixed layers (Zimmerman et al. 2000; Pickart et al. 2002). An expanded view of such smaller-scale homogenized sublayers within a convective layer is shown in figure 7 , where the sublayers are highlighted by vertical segments to aid the eye. To calculate the spice, the equation of state of sea water was linearized with respect to small departures from the typical values $\theta_{*}=3.15^{\circ} \mathrm{C}$ and $S_{*}=34.806$ PSU observed within the mixed layer: $\theta^{\prime}=\theta-\theta_{*}, S^{\prime}=S-S_{*}$. The ranges of $\theta$ and $S$ plotted in figure 7 are chosen in such a way that they contribute equally to the density and 
spice anomalies. Thus, we clearly see that the contributions of $\theta^{\prime}$ and $S^{\prime}$ to the density field are indeed largely compensated: the variability of $\sigma_{0}$ is reduced, while the spice enhances and dramatically reveals the steps. Such a steplike structure was observed at many hydrographic stations within a convective region (R. S. Pickart, personal communication). These sublayers having thickness of 50 to $100 \mathrm{~m}$ are not microstructure and their origin is currently unknown. From a dynamical point of view, their existence in itself is a surprise: it is very unlikely that these layers are quasi-horizontal, for they would not survive strong vertical velocities in an actively convecting region. Our hypothesis is that these sublayers may be analogous to the tilted 'ink walls' and represent internal small-scale tilted structures within a plume, as suggested by the laboratory experiments.

Furthermore, thinking in an opposite way, the rotational control of the turbulent convective motions sets the limit on homogenization of waters in the mixed layer by virtue of (6.4).

The existence of such tilted structures can be used as a fingerprint of a certain phase of convection. In particular, it is known that after convection stops, the restratification of the 'chimney' happens very rapidly (within a month rather than on a seasonal scale). What is the role of tilted structures in this process? How long do they survive after convection stops? Does restratification occur by means of quasi-horizontal layered intrusions or by vertically coherent tilted structures sheared by the quasi-horizontal flow? Do we have to reinterpret some observations of horizontal 'layers' as being tilted structures? To definitely assert that the observed sublayers represent a tilted structure, higher-resolution data obtained (with horizontal spacing of about $100 \mathrm{~m}$ ) in an actively convecting region are necessary; no such observations are known to the author. Thus, more studies of the formation of the tilted structure both in the field and in the laboratory are required.

\section{Conclusions}

The motivation for this work came from discussing the nature of convective motions in a rotating fluid with a number of scientists. The central question was in which direction the plume will spread in the case of a finite $\alpha$. Dr Alan Faller commented that in his old laboratory experiments, which were performed in a rotating tank having the shape of a paraboloid, he could not obtain a definite answer because the view was oblique and greatly distorted by refraction. It is the use of the centrifuge with the tank placed at a large distance from the centre of a turntable that allows us to properly model in a laboratory the geophysically relevant case of a finite angle between the gravitational force and the axis of rotation, with both vectors fixed in the rotating reference frame, and to obtain three simultaneous undistorted views of convection.

Based on the analysis of forces acting on a solid ellipsoid moving through rotating fluid, we conclude that, if we think of the plume as an ensemble of ellipsoids or similar coherent structures, then the balance of the buoyancy, Coriolis forces and hydrodynamic reaction due to inertial wave generation explains the tendency of the sinking plume to develop in the intermediate direction between $\boldsymbol{g}_{e}$ and $\boldsymbol{\Omega}$ and also to shift 'eastward'. The misalignment between $\boldsymbol{g}_{e}$ and $\boldsymbol{\Omega}$ also contributes to enhanced turbulent mixing and development of tilted structures due to the breaking of symmetry and generating off-axis horizontal motions. We argue that the conditions at the sites of ocean deep convection are favourable for the development of tilted structures because of the smallness of the Rossby number and an extreme homogenization of 
the mixed layer. We encourage the observational efforts aimed at detecting such tilted structures.

The author is very grateful to laboratory assistant John Salzig, who rebuilt and reinforced an old turntable and built most of the centrifuge. Special thanks are due to Barbara Gaffron for proofreading the manuscript. Stimulating discussions with Sergei Dikarev, Fiamma Straneo, Bob Pickart, Joe Pedlosky, David Loper, Alan Faller and John Marshall were especially fruitful. This work was supported by a grant from The Andrew W. Mellon Foundation Endowed Fund for Innovative Research and by the National Science Foundation grant OCE-0116910. This manuscript is Woods Hole Oceanographic Institution Contribution Number 10446.

\section{REFERENCES}

Batchelor, G. K. 1967 An Introduction to Fluid Dynamics. Cambridge University Press.

Boubnov, B. M. \& Golitsyn, G. S. 1995 Convection in Rotating Fluids. Kluwer.

Bush, J. W. M., Stone, H. A. \& Bloxham, J. 1995 Axial drop motion in rotating fluids. J. Fluid Mech. 282, 247-278.

Bush, J. W. M., Stone, H. A. \& TAnzosh, J. P. 1994 Particle motion in rotating viscous fluids: historical survey and recent developments. Current Topics Phys. Fluids 1, 337-355.

Busse, F. H., Hartung, G., Jaletzky, M. \& Sommermann, G. 1998 Experiments on thermal convection in rotating systems motivated by planetary problems. Dyn. Atmos. Oceans 27, $161-174$

Chu, P. C. \& Gascard, J. C. (Ed.) 1991 Deep Convection and Deep Water Formation in the Oceans. Elsevier.

Emanuel, K. A. 1988 Observational evidence of slantwise convective adjustment. Mon. Weather Rev. 116, 1805-1816.

Fernando, H. J. S., Chen, R.-R. \& Ayotte, B. A. 1998 Development of a point plume in the presence of background rotation. Phys. Fluids 10, 2369-2383.

FletcheR, R. I. 1972 The apparent field of gravity in a rotating fluid system. Am. J. Phys. 40, 959-965.

Greenspan, H. P. 1990 The Theory of Rotating Fluids. Breukelen.

Helfrich, K. R. 1994 Thermals with background rotation and stratification. J. Fluid Mech. 259, 265-280.

Hide, R., Ibbetson, A. \& Lighthill, M. J. 1968 On slow transverse flow past obstacles in a rapidly rotating fluid. J. Fluid Mech. 32, 251-272.

Lilly, J. M. \& Rhines, P. B. 2002 Coherent eddies in the Labrador Sea observed from a mooring. J. Phys. Oceanogr. 32, 585-598.

Lilly, J. M., Rhines, P. B., Visbeck, M., Davis, R., Lazier, J. R. N., Schott, F. \& Farmer, D. 1999 Observing deep convection in the Labrador Sea during winter 1994/95. J. Phys. Oceanogr. 29, 2065-2098

LoNG, R. R. 1954 Note on Taylor's 'ink walls' in a rotating fluid. J. Met. 11, 247-249.

LOPER, D. E. 2001 On the structure of a Taylor column driven by a buoyant parcel in an unbounded rotating fluid. J. Fluid Mech. 427, 131-165.

Macdonald, A. \& WunsCH, C. 1996 A global estimate of the ocean circulation and heat fluxes. Nature 382, 436-439.

Mamayev, O. I. 1975 Temperature-Salinity Analysis of World Ocean Waters. Elsevier (transl. from Russian by R. J. Burton).

Marshall, J. \& Schott, F. 1999 Open-ocean convection: observations, theory, and models. Rev. Geophys. 37, 1-64.

MaXworthy, T. \& Narimousa, S. 1994 Unsteady, turbulent convection into a homogeneous, rotating fluid, with oceanographic applications. J. Phys. Oceanogr. 24, 865-887.

Meng, H., Estevadeordal, J., Gogineni, S. \& Goss, L. 1998 Holographic flow visualization as a tool for studying three-dimensional coherent structures and instabilities. J. Visualization 1, $133-144$ 
Mollo-Christensen, E. 1972 Flow instabilities. (Videorecording). Text: Illustrated Experiments in Fluid Mechanics, pp. 113-120. National Committee for Fluid Mechanics. Cambridge, MA: Educational Development Center.

Morton, B. R., TAYLOR, G. \& TuRner, J. S. 1956 Turbulent gravitational convection from maintained and instantaneous sources. Proc. R. Soc. Lond. A 234, 1-23.

Munk, W. 1981 Internal waves and small scale processes. In Evolution of Physical Oceanography: Scientific Surveys in Honor of Henry Stommel (ed. B. A. Warren \& C. Wunsch), pp. 264-291. MIT Press, Cambridge, MA.

Pickart, R. S., Torres, D. J. \& Clarke, R. A. 2002 Hydrography of the Labrador Sea during active convection. J. Phys. Oceanogr 32, 428-457.

Proudman, J. 1916 On the motion of solids in liquids possessing vorticity. Proc. R. Soc. Lond. A 92, 408-424.

Riemenschneider, U. 2002 Ball release experiment in a centrifuge. In Bounds on Turbulent Transport. 2002 Summer Study Program in Geophysical Fluid Dynamics. Tech. Rep., pp. 194-213 (available at http://gfd.whoi.edu/proceedings/2002/PDF/Reimenschneider.pdf).

Schott, F., Visbeck, M. \& Fischer, J. 1993 Observations of vertical currents and convection in the central Greenland Sea during the winter of 1988/89. J. Geophys. Res. 98, 14 401-14 421.

Steffen, E. L. \& D'Asaro, E. A. 2002 Deep convection in the Labrador Sea as observed by Lagrangian floats. J. Phys. Oceanogr. 32, 475-492.

Stewartson, K. 1953 On the slow motion of an ellipsoid in a rotating fluid. Q. J. Mech. Appl. Maths 6, 141-162.

Straneo, F., Kawase, M. \& Riser, S. C. 2002 Idealized models of slantwise convection in a baroclinic flow. J. Phys. Oceanogr. 32, 558-572.

TAYLOR, G. I. 1916 Motion of solids in fluids when the flow is not irrotational. Proc. R. Soc. Lond. A 93, 99-113.

TAYLOR, G. I. 1921 Experiments with rotating fluids. Proc. R. Soc. Lond. A 100, 114-121.

TAYLOR, G. I. 1922 The motion of a sphere in rotating liquid. Proc. R. Soc. Lond. A 102, 180-189.

TURNer, J. S. 1962 The 'starting plume' in neutral surroundings. J. Fluid Mech. 13, 356-371.

VAughn, S. L. \& LEAman, K. D. 1995 The role of small-scale cells in the Mediterranean convection process. J. Phys. Oceanogr. 25, 2423-2436.

Veronis, G. 1972 On properties of seawater defined by temperature, salinity, and pressure. J. Mar. Res. 30, 227-255.

Zatsepin, A. G. 2001 On peculiarities and similarities of the coherent structure formation in stratified and rotating fluid. In Turbulent Mixing in Geophysical Flows (ed. P. F. Linden \& J. M. Redondo), pp. 277-300. CIMNE.

Zimmerman, S., McKee, T. K., Pickart, R. S. \& Smethie, W. M. 2000 Knorr 147 Leg V hydrographic data report: Labrador Sea deep convection experiment. Woods Hole Oceanogr. Inst. Tech. Rep. WHOI-2000-05. 